\title{
Modelling, computation and analysis on combustion of explosives
}

\author{
S. SAID ${ }^{1}$, F. T. SMITH ${ }^{1}$ and J. P. CURTIS ${ }^{1,2}$ \\ ${ }^{1}$ Department of Mathematics, University College London, London, WC1H OAY, UK \\ email: s.said.17@ucl.ac.uk \\ ${ }^{2}$ AWE Aldermaston, Reading, RG7 $4 P R, U K$
}

(Received 24 March 2020)

\begin{abstract}
When an explosive burns, gaseous products are formed as a result. The interaction of the burning solid and gas is not well understood. More specifically, the process of the gaseous product heating the explosive is yet to be explored in detail. The present work sets out to fill some of that gap using mathematical modelling: this aims to track the temperature profile in the explosive. The work begins by modelling single step reactions using a simple Arrhenius model. The model is then extended to include three step reaction. An alternative asymptotic approach is also employed. There is close agreement between results from the full reaction-diffusion problem and the asymptotic problem.
\end{abstract}

Key Words: Reaction-diffusion, combustion, thermodynamics.

2010 Mathematics Subject Classification: 35K57, 80A25 (Primary); 74A15 (Secondary)

\section{Introduction}

High explosives (HEs) provide a low mass source of massive energy release, but this stored energy can pose a major hazard and even cause disaster if accidentally released. There is a long and sad history of serious accidents that have resulted from both mechanical and thermal abnormal and unexpected loadings of diverse severities. Therefore, safety is paramount in the handling and storage of explosives. Understanding the circumstances in which an explosive can ignite, burn and detonate is essential if we are to predict the severity of likely hazards and understand the associated risks [1, [2].

The high cost and danger of experimentation has necessitated the modelling of scenarios of potential danger. The great flexibility and power of hydrocode packages implementing finite element and difference methods [3, 4] has made their use the natural choice to address this need. However, the use of these codes has often revealed that localised effects arise that are hard to resolve with standard computation meshes; often shear bands or local regions of extreme heating are observed. The question thus arose: could mathematical analysis and/or new sophisticated adaptive methods offer further advances both in understanding the governing physics and chemistry, and in achieving improved computational models? 
Let us attempt to summarise our current understanding of the processes happening in a reacting high explosive. HEs may be distinguished from other types by their capability to detonate rather than burn if sufficient stimulus is applied to them. Under lesser stimuli, they may burn [5] and the resulting reaction can diminish, remain steady, or grow to more violent deflagration or even to detonation, where the stored explosive energy is released in times typically of the order of nanoseconds. What is governing these very diverse outcomes?

It is established that, when a $\mathrm{HE}$ is subject to significant heating as a result of either mechanical dissipation caused by accidental severe deformation or direct heating from a heat source, it begins to react. The solid material reacts, i.e. burns to form high pressure gaseous products $[6,7,8,9,22]$. As the reaction proceeds more and more gas is formed. The porosity of the explosive increases and as more and more surface area becomes exposed the reaction can accelerate and propagate with the increasing porosity and permeability 10 until all the explosive is consumed or until some mechanism releases the pressure and the reaction is quenched. Violent reaction or even disastrous detonation can be achieved in some cases. We believe that it is fair to say that the interplay between the burning (and thereby disintegrating) solid matrix and gaseous products is still ill-understood. Plainly there are confined locations where flames may be interacting with flames from nearby surfaces, probably in highly complex ways.

Baer and Nunziato 11 and subsequent workers have explored the two-phase problem of reacting solid and gaseous products from a macroscopic continuum viewpoint, but detailed treatments of the internal burning process and of how the hot gas heats the explosive up are lacking. The problem is compounded by the complexity of modern heterogeneous explosives in which crystals of pure HE are embedded in polymer binders, which themselves can be reactive. The creation and propagation of flames in this type of explosive have not been modelled in detail; the computational costs would be prohibitive even were this possible. Therefore, burn models in current use are generally empirically based macroscopic models rather than being based upon first principles. Their calibration often depends on the experiment or geometry being modelled. That being so, we asked ourselves the question: Would it be possible to gain deeper understanding of the physical processes at work in real HEs by looking at scenarios with idealised geometries lending themselves to analytical modelling?

We believe this is a question worthy of attention and the present paper reports one research line taken in attempting to answer. We additionally comment that the recent advances in additive manufacturing of explosives provide enhanced motivation for the investigation of idealised explosive geometries, which are becoming realisable in practice while being potentially more amenable to mathematical modelling [12, 13, 14, 15]. The modelling to be used herein is based on a continuum assumption.

Our aims, then, include gaining further insight, providing comparisons or alternatives for direct computation and adding to the portfolio of methodologies available in this area 16. Section 2 describes the model of interaction evolving between thermal diffusion effects in one spatial dimension and a single reactant $7,17,12,18,15$, along with a computational study. Here reference is made to ODTX (One dimensional time to explosion methods) 19, 11, 20, 17 and HMX (octogen explosive) for which the values of certain parameters in the model turn out to be significantly small or large. This leads on to an in- 


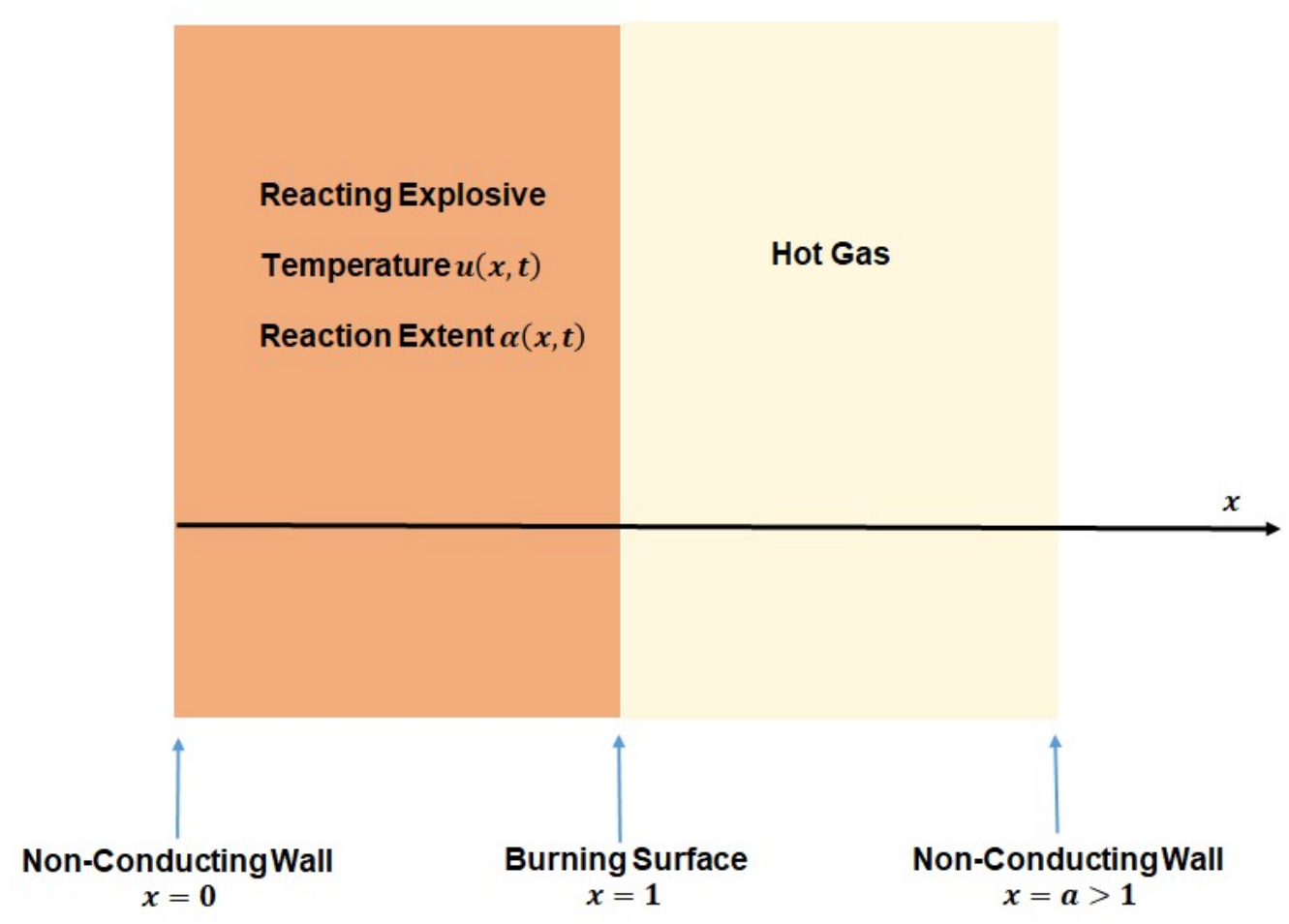

Figure 1. Schematic of the solid explosive (left) and hot gas (right).

vestigation of asymptotic properties presented in Section 3 accompanied by comparisons with the direct computations. Section 4 then addresses the evolution between thermal diffusion and three reactants 21] computationally followed by corresponding asymptotic analysis and comparisons given in Section 5 . Section 6 provides the conclusion, including a further discussion of the continuum assumption.

\section{One-reactant problem and computational properties}

The problem area of concern here arises as follows. A simple configuration is considered, with a slab of solid explosive confined at one end with a burning surface at the other end. A layer of hot gas is adjacent to the burning surface, itself confined by a non-conducting wall opposite to the burning surface. See Figure 1. When the hot gas is assigned a temperature value that is in the vicinity of the activation temperature for the explosive, this acts as a trigger for the ignition of the explosive. Classical heat conduction 27, 28, 23 in the explosive is one feature with a significant role in the model here.

We then enhance the model by considering the effects of adding a reaction term to the heat conduction equation 29]. To begin modelling the reaction-diffusion process, we consider the simple and well-known Arrhenius reaction equation [1. 
The Arrhenius equation 21] is used to calculate the effect of a change in temperature on reaction rates. It is commonly used to calculate chemical reactions, particularly in heat induced problems. The combustion of explosives depends heavily on chemical processes which take place. Heating and impact both can trigger a reaction process which leads to significant burning, and they are the main two reasons why an explosive combusts. Once the temperature reaches a certain level, commonly known loosely as the critical temperature, the reaction process becomes significantly large. When the reaction has started, the speed of the reaction increases as the temperature increases. The Arrhenius reaction equation has been used in several explosives models including [7] and is given by

$$
k=A \exp (-E / R u),
$$

where $k$ is the reaction rate constant, $\mathrm{u}$ is the temperature, $E$ is the activation energy required for the reaction, $R$ is the universal gas constant and $A$ is the frequency factor, which is also known as the pre-exponential constant.

Following the works of 7, 30, 29, for example, we consider a variation of (2.1). Namely, we consider the reaction $\alpha$ with rate such that $k \rightarrow \frac{\frac{\partial \alpha}{\partial t}}{(1-\alpha)}$ which mimics the general form (2.1) but varies with time $t$. The reaction rate is restricted by the term $(1-\alpha)$, which represents the fraction of unreacted material remaining. In other words $0 \leqslant \alpha(x, t) \leqslant 1$ for all spatial positions $x \in D$ and $t \in T$ where $\alpha=0$ is the initial state (unreacted) and $\alpha=1$ is the final state (fully reacted). Here $T=\left[0, T_{\max }\right]$, where $T_{\max }$ is the period of time over which we model the event, and $D$ is the bounded spatial domain, which can be taken as $[-1,1]$ without loss of generality by working with $x / a$ and $\kappa / a^{2}$ for a domain $[-a, a]$, where the diffusion coefficient $\kappa$ is defined below. We use the reflected geometry to avoid the requirement to impose a zero flux condition for simplicity. However, if we did adhere to $x \geqslant 0$ we would need to impose that condition, but might reduce the computational time.

We now consider the reaction-diffusion PDE

$$
\rho c_{v} \frac{\partial u}{\partial t}=\kappa \frac{\partial^{2} u}{\partial x^{2}}+\rho \Omega \frac{\partial \alpha}{\partial t}
$$

with the reaction term given by

$$
\frac{\partial \alpha}{\partial t}=A(1-\alpha) \exp \left(-\frac{E}{R u}\right),
$$

where the constant $\Omega$ is the heat of reaction. Equations $\sqrt{2.2}$ and $(2.3)$ combine to form a non-linear coupled system of PDEs for $u(x, t)$ and $\alpha(x, t)$.

Here $\rho, c_{v}, \kappa, \Omega, R, E$ and $A$ are dimensional parameters with realistic values and units listed in Table 1. The typical boundary and initial conditions are

$$
\begin{array}{r}
u(-1, t)=u(1, t)=B, \quad \text { for } 0<t \leqslant T_{\max }, \\
u(x, 0)=C, \quad \text { for } x \in D,
\end{array}
$$

where quite realistic temperature values are $B=570, C=293$ say. This spatially symmetric scenario with constant boundary and initial temperatures is to be generalized later, whereas the initial value of $\alpha$ is taken to be zero in all the present studies.

Given the extremely large dimensional values appearing in Table 1 and in the conditions 
Table 1. Physical parameters used in model.

\begin{tabular}{lcl}
\hline \hline Parameter with units & Symbol & Value \\
\hline Conductivity $(\mathrm{W} / \mathrm{m} / \mathrm{K})$ & $\kappa$ & 0.44 \\
Specific Heat $(\mathrm{J} / \mathrm{kg} / \mathrm{K})$ & $c_{v}$ & 1255.0 \\
Density $\left(\mathrm{kg} / \mathrm{m}^{3}\right)$ & $\rho$ & 1800.0 \\
Heat of Reaction $(\mathrm{J} / \mathrm{kg})$ & $\Omega$ & $5.0208 e 6$ \\
Molar Gas Constant $(\mathrm{J} / \mathrm{mol} / \mathrm{K})$ & $R$ & 8.314 \\
Activation Energy $(\mathrm{J} / \mathrm{mol})$ & $E$ & $2.2 e 5$ \\
Pre-exponential Constant $\left(\mathrm{s}^{-1}\right)$ & $A$ & $5.011872336 e 19$ \\
Wall temperature ${ }^{\circ} K$ & $B$ & 570 \\
Initial solid temperature ${ }^{\circ} K$ & $C$ & 293 \\
\hline \hline
\end{tabular}

$2.4 a 2.4 b$ we turn to a non-dimensional form of the governing equations and conditions and note a need for caution concerning numerical results at this stage. To deal with the parameters, then, we introduce the non-dimensional variables $\bar{t}$ and $\bar{u}$ that satisfy

$$
t=A^{-1} \bar{t}, \quad u=\frac{E}{R} \bar{u}
$$

having recognised the fact that $A$ is likely to be responsible for main changes in $u$ and $\alpha$ happening on different time scales and that $\frac{E}{R}$ is relatively large. There is no need to scale $\alpha$ since it is already non-dimensional and $\mathcal{O}(1)$.

The substitutions lead to the non-dimensional system of PDEs

$$
\begin{aligned}
& \frac{\partial \bar{u}}{\partial \bar{t}}=\bar{\kappa} \frac{\partial^{2} \bar{u}}{\partial x^{2}}+\bar{\Omega} \frac{\partial \alpha}{\partial \bar{t}}, \\
& \frac{\partial \alpha}{\partial \bar{t}}=(1-\alpha) \exp \left(-\frac{1}{\bar{u}}\right),
\end{aligned}
$$

where from Table 1 the non-dimensional parameters present now are $\bar{\kappa}:=\frac{\kappa}{\rho c_{v} A} \approx$ $10^{-25} \ll 1$, and $\bar{\Omega}:=\frac{\Omega R}{c_{v} E} \approx 0.15$. The boundary and initial conditions also need scaling and are given by

$$
\begin{aligned}
\bar{u}(-1, t) & =\bar{u}(1, t)=\bar{B}, \quad \text { for } 0<\bar{t} \leqslant T_{\max } \\
\bar{u}(x, 0) & =\bar{C}, \quad \text { for } x \in D
\end{aligned}
$$

where $\bar{B}:=\frac{R B}{E} \approx 0.02$ and $\bar{C}:=\frac{R C}{E} \approx 0.01$. We note that the initial condition for $\alpha$ remains unchanged of course and that quite extreme parameter values are still present, particularly the $\bar{\kappa}$ value.

Numerical solutions were sought first, using a semi-implicit scheme of second-order accuracy in $x, t$. This adopts three-point backward differencing in $t$ and three-point 
centred differencing in $x$. The discretisation replaces 2.2 - 2.3 by

$$
\begin{array}{r}
-\mu U_{j-1, i+1}+(3+2 \mu) U_{j, i+1}-\mu U_{j+1, i+1}=4 U_{j, i}-U_{j, i-1}+\ldots \\
\cdots+2 \Delta t \frac{A \Omega}{c_{v}}\left(1-2 \alpha_{j, i}+\alpha_{j, i-1}\right) \exp \left(-\frac{E}{R\left(2 U_{j, i}-U_{j, i-1}\right)}\right), \\
\alpha_{j, i+1}=\frac{2 A \Delta t \exp \left(\frac{-E}{R U_{j, i+1}}\right)+4 \alpha_{j, i}-\alpha_{j, i-1}}{3+2 A \Delta t \exp \left(\frac{-E}{R U_{j, i+1}}\right)},
\end{array}
$$

and it acts similarly on the form 2.6a, $2.6 b$. Here $i, j$ refer to time $t$ and space $x$ respectively, with grid sizes $\Delta t, \Delta x$, the arrays $U_{j, i}, \alpha_{j, i}$ represent discretised $u, \alpha$ and $\mu=r \frac{2 \Delta t}{(\Delta x)^{2}}$, where $r=\frac{\kappa}{\rho c_{v}}$. We remark that, to keep the scheme quasi-linear at each new time step, lagging of terms in the exponential effects in $2.8 a), 2.8 b)$ is present. For example in the exponential effect in $2.8 a$ the expression $\left(2 U_{j, i}-U_{j, i-1}\right)$ is used in place of $U_{j, i+1}$ and preserves the desired second-order accuracy. The parameter values involved are of much interest. The fairly realistic values in practice shown in Table 1 are potentially quite extreme values; indeed we investigate the influences of a quite wide range of values of the parameters below.

In fact cautiously obtaining and then considering the computational solutions is felt to be very desirable for mild, less mild and realistic cases. We defer further discussion of the realistic cases such as in Table 1 until Section 6. Figures 2 2 show the numerical results for mild cases. They exhibit a number of interesting features. There is analytically a classical similarity solution 31 with spatial thickness of order $t^{1 / 2}$ holding in each thin edge layer at small time astride each wall, a property which is captured satisfactorily by the current numerical scheme. One can see evidence of thin edge layers emerging also as $\kappa$ or $\bar{\kappa}$ is decreased. These layers however continue to apply over a considerable time range. The numerical results further produce overshoots in temperature which become more pronounced and linger more (see Figure 6 ) as $\bar{\kappa}$ is decreased for example, whereas $\alpha$ increases monotonically towards unity in every case. We see in addition some pronounced differences in the apparent time scales typical of the temperature and $\alpha$ in the results. These are to be considered analytically later. The results in Figures 26 are only showing spatially symmetric cases but non-symmetric ones, presented in Figure 7 display similar trends.

The accuracy of the numerical solutions was checked as in 32 . We found the realistic case with the values of Table 1 to be a very difficult one to compute reliably in view of, for example, a sensitivity observed in the results for $\alpha$ as the spatial step was varied. In contrast, when we use milder parameter values such as $E=10$ and $A=200$ with the boundary and initial conditions $(2.4 a)-(2.4 b)$ with $B=45, C=15$ then the finite difference approximation of $\alpha$ is insensitive to the same choices of spatial step. The profiles of these solutions are given in Figure 4 A further set of results for even milder values of the parameters is presented in Figures 5, 6. pointing to the use of asymptotic analysis below.

To address the very small parameter $\bar{\kappa}$ that features in the scaled PDE, we add a different approach. It can be shown through asymptotic expansions of $\bar{u}$ and $\alpha$ that by equating the coefficients of like powers of $\bar{\kappa}$ and taking $\mathcal{O}(1)$ terms only, the term $\frac{\partial^{2} \bar{u}}{\partial x^{2}}$ is 
(a)

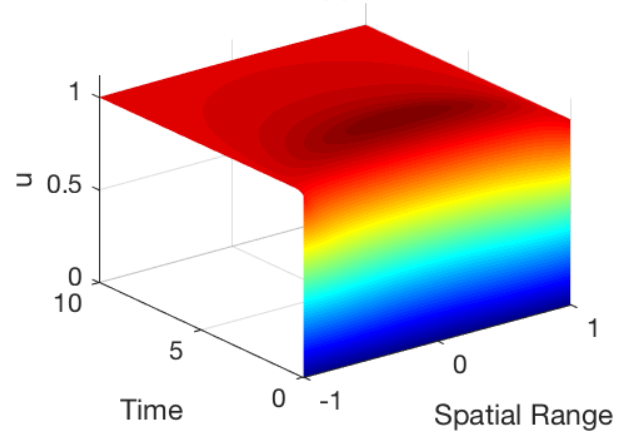

(c)

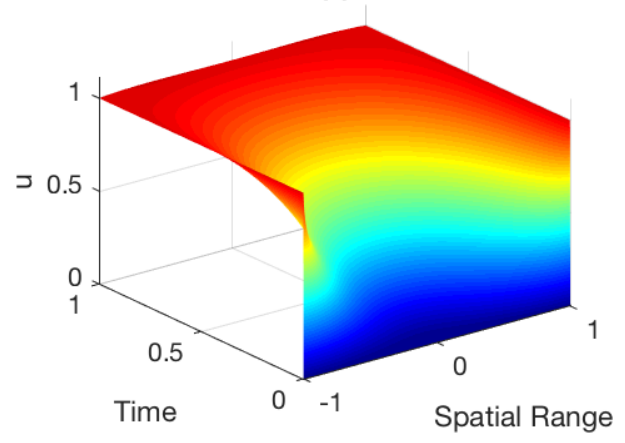

(b)

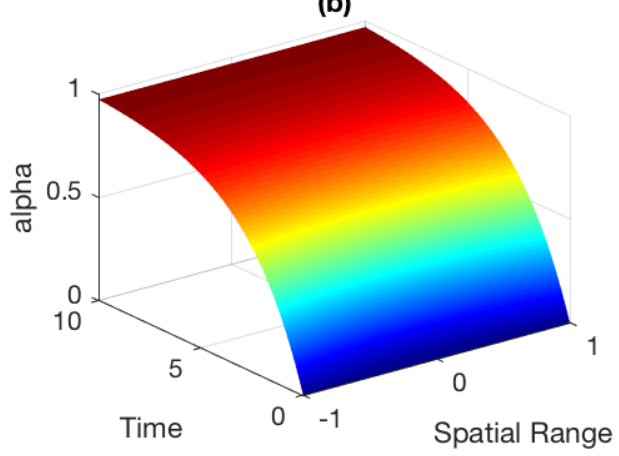

(d)

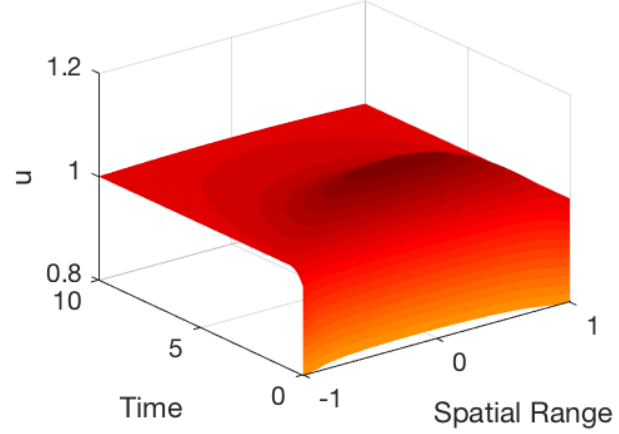

Figure 2. The finite difference solution to $u$ (figure (a)) and $\alpha$ (figure (b)) in the coupled system of PDEs (2.2) and (2.3) using a second-order implicit scheme. We chose $T_{\max }=10$ with $\Delta x=0.05$ and $\Delta t=0.01$. Here $\rho, c_{v}, \kappa, \Omega, A, E, R$ are set to unity. Also $B=45$ and $C=15$ (in non-dimensional terms, $\bar{\Omega}=1, \bar{\kappa}=1, \bar{B}=45$ and $\bar{C}=15$ ). On the bottom left (figure (c)) we plot the solution for small time $t=0$ to $t=1$ to show the rise of the temperature, $u$. Notice how the inclusion of a reaction term causes the temperature profile to surpass the temperature value at the boundary before plateauing (figure (d)).

unlikely to have any substantial effect for a long time, except near the boundaries, since the reaction term dominates. Hence, asymptotically, the problem can now be viewed as the interaction of two problems: a problem in thin wall layers at both ends of the domain and a problem in a core.

The view just mentioned in regard to the emergence of two problems accompanied by distinct regions is especially supported by the numerical results for the single-reactant cases shown in Figures 5 77, where Figure 7 admits spatial nonsymmetry. The results in the figures, which are for parameter values even milder than those introduced in Figures 2,4. confirm clearly the appearance of a core in the majority of the domain and thin wall layers near the boundaries even for values of $\kappa$ for example that are small but, in a sense, not extremely so. This suggests that an asymptotic approach (as described below) will be fruitful over a wide range of the parameter space. 

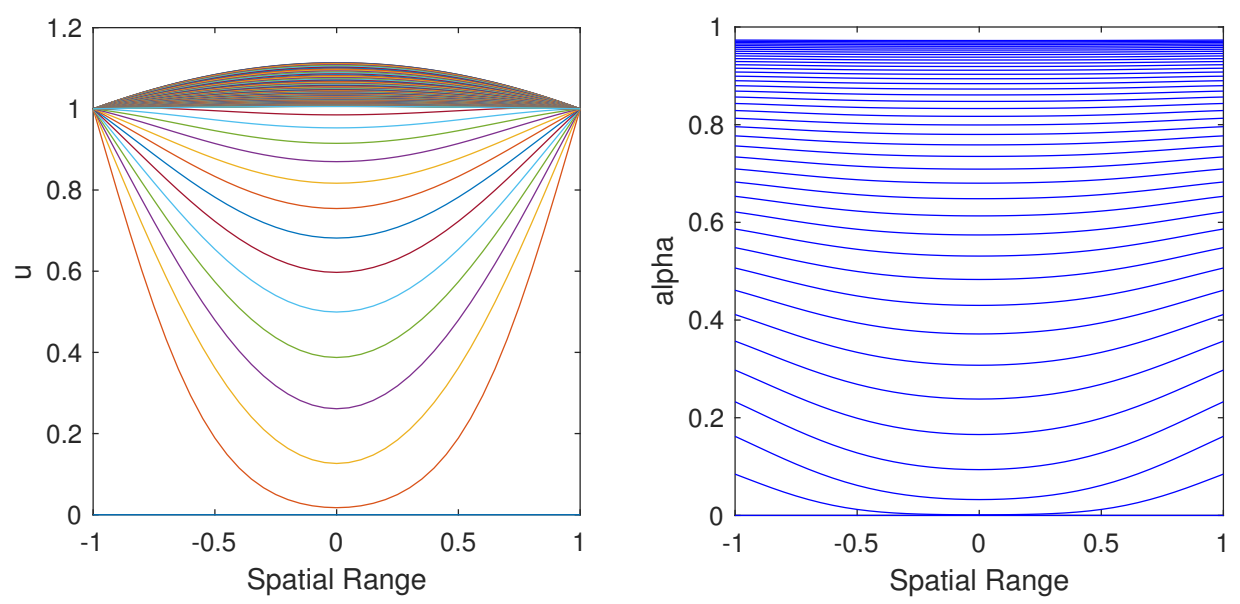

Figure 3. Solutions to $u$ and $\alpha$ over the spatial range $x$ for fixed values of $t$. Here we have used the same parameters and temperature conditions as in Figure 2

\section{Asymptotic analysis and comparisons}

Guided by the numerical solution features above we seek extra insight by taking an asymptotic approach that in principle handles effectively the extreme parameter values. A core region covering most of the domain can be anticipated along with thin wall layers close to the boundaries. That distinction in spatial scale is found to persist for a considerable amount of time before change occurs. The initial condition on the temperature is taken to be constant for now but this is generalised later on.

\subsection{Effects of $\bar{\kappa}$}

The main evidently small parameter is $\bar{\kappa}$. Treating it as an asymptotically small parameter leads to two major time scales appearing as follows. The first time scale has $\bar{t}$ of $\mathcal{O}(1)$. Here in the core where $-1<x<1$ the variables $\bar{u}, \alpha$ are expected to be of order unity and so the expansions

$$
\begin{aligned}
\bar{u} & =u_{c}+\ldots, \\
\alpha & =\alpha_{c}+\ldots
\end{aligned}
$$



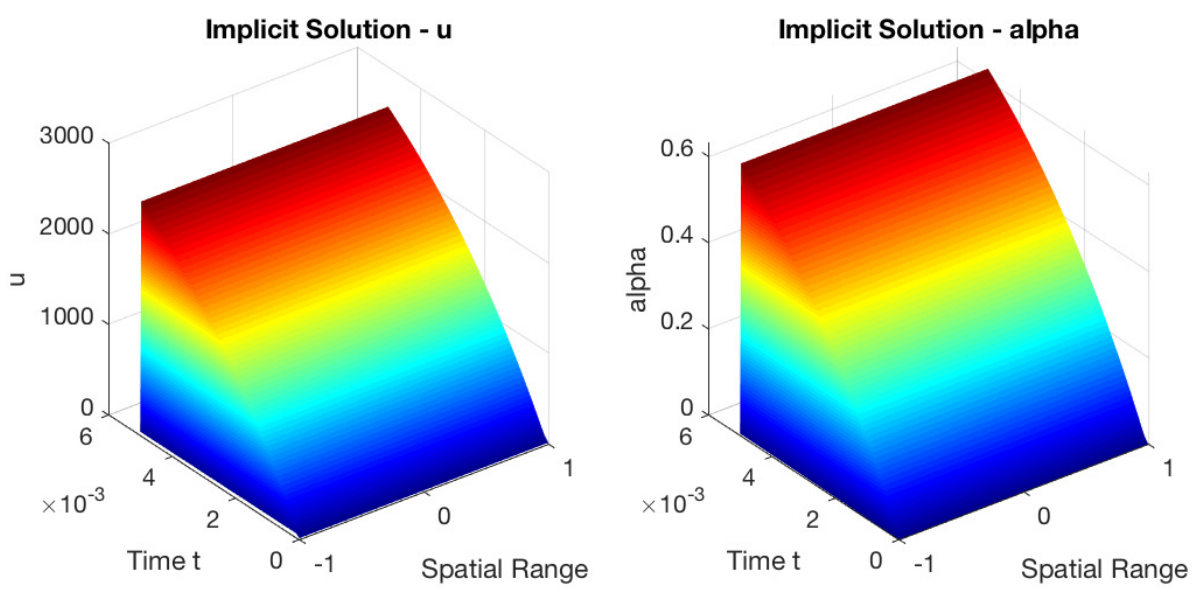

Figure 4. The numerical finite difference solutions to $u$ (left) and $\alpha$ (right) satisfying the PDEs 2.2 - 2.3 using a second-order implicit scheme with mild physical parameters. Here $\rho=1800, c_{v}=1255, \kappa=0.44, \Omega=5.0108 \times 10^{6}, A=200, E=10, R=8.314$, $B=45$ and $C=15$. In non-dimensional terms, $\bar{\Omega} \approx 3319.505, \bar{\kappa} \approx 9.7 \times 10^{-10}, \bar{B} \approx 37.413$ and $\bar{C} \approx 12.471$.

are called for. Terms of equal orders in the non-dimensional governing equations 2.6 then yield a reduced system for the leading order quantities, namely

$$
\frac{\partial \bar{u}_{c}}{\partial \bar{t}}=\bar{\Omega} \frac{\partial \alpha_{c}}{\partial \bar{t}},
$$

where

$$
\frac{\partial \alpha_{c}}{\partial \bar{t}}=\left(1-\alpha_{c}\right) \exp \left(-\frac{1}{\bar{u}_{c}}\right)
$$

The leading equation above admits the simple result that $\bar{u}_{c}-\bar{\Omega} \alpha_{c}$ is a function of $x$ only but the initial condition of a constant $u$ across the domain then establishes that function is constant. Therefore applying the initial conditions that $\bar{u}_{c}(x, 0)=\bar{C}$ and $\alpha_{c}(x, 0)=0$ yields the relation

$$
\bar{u}_{c}(x, \bar{t})=\bar{\Omega} \alpha_{c}(x, \bar{t})+\bar{C},
$$

for all $\bar{t}$ of $\mathcal{O}(1)$. Hence equation $(3.3)$ becomes one for $\alpha_{c}$ alone,

$$
\frac{d \alpha_{c}}{d \bar{t}}=\left(1-\alpha_{c}\right) \exp \left(-\frac{1}{\bar{\Omega} \alpha_{c}+\bar{C}}\right) .
$$

Note that since the initial condition $\alpha_{c}(x, 0)=0$ is independent of $x,(3.2)$ as it stands is also independent of $x$ and essentially represents a non-linear ODE for $\alpha_{c}$ that is valid for $-1<x<1-$. We remark in passing here that $\mathrm{x}$-dependence in the initial conditions at $t=0$ which can be reflected in $\bar{C}$ in (3.4), 3.5) and similarly $\alpha(x, 0)$ being given functions of $x$ will be discussed later. Clearly the diffusive term involving a double $\mathrm{x}$-derivative is 

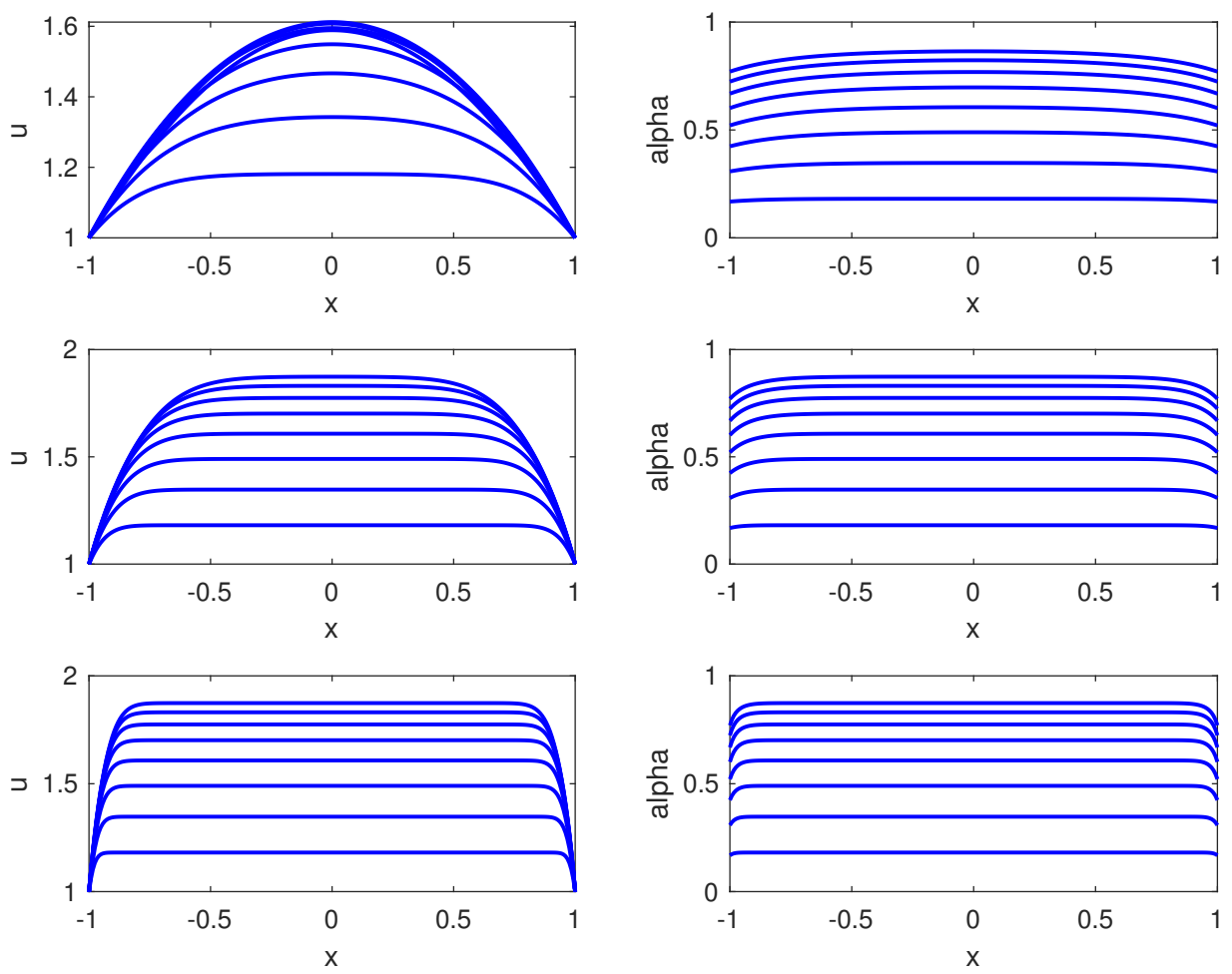

Figure 5. The numerical results for $u$ and $\alpha$ satisfying equations 2.2 - 2.3 with very mild parameter values $E=R=A=\rho=\Omega=c_{v}=B=C=1$ and $\kappa=0.1,0.01,0.001$ (top to bottom). In non-dimensional terms, $\bar{\Omega}=1, \bar{B}=1, \bar{\kappa}=0.1,0.01,0.001$ (top to bottom) and $\bar{C}=1$.

negligible in the core at this level and no boundary condition is applied. The ODE (3.5) is non-trivial to solve and so we use ODE45 in MATLAB to integrate numerically. The core reaction $\alpha_{c}$ may then be substituted back into (3.4) to determine the leading order temperature $\bar{u}_{c}$ in the core.

In Figure 8 we plot the core solutions $\bar{u}_{c}$ and $\alpha_{c}$ that satisfy 3.3 and 3.2 , respectively, using the mild parameter values $E=10$ and $A=200$ as well as $C=15$ which is required for the initial condition of $\bar{u}_{c}$. The asymptotic analysis is still formally valid using these mild parameters since the corresponding value of $\bar{\kappa}$ is still exceptionally small. We use mild parameters for now so that in a sense we may check (see Figure 9 the asymptotic analysis against the finite difference solutions given in Section 2 before proceeding to employ the full parameter values of Table 1. Further details on the effects of treating $\bar{\Omega}$ as a large parameter are given in a subsection below. It is worth noting that the characteristics of the core with mild parameters and physical parameters are likely to be different because the parameters change not only the boundary layer but also the behaviour of the core. Results for other parameter values are given in the next subsection. 

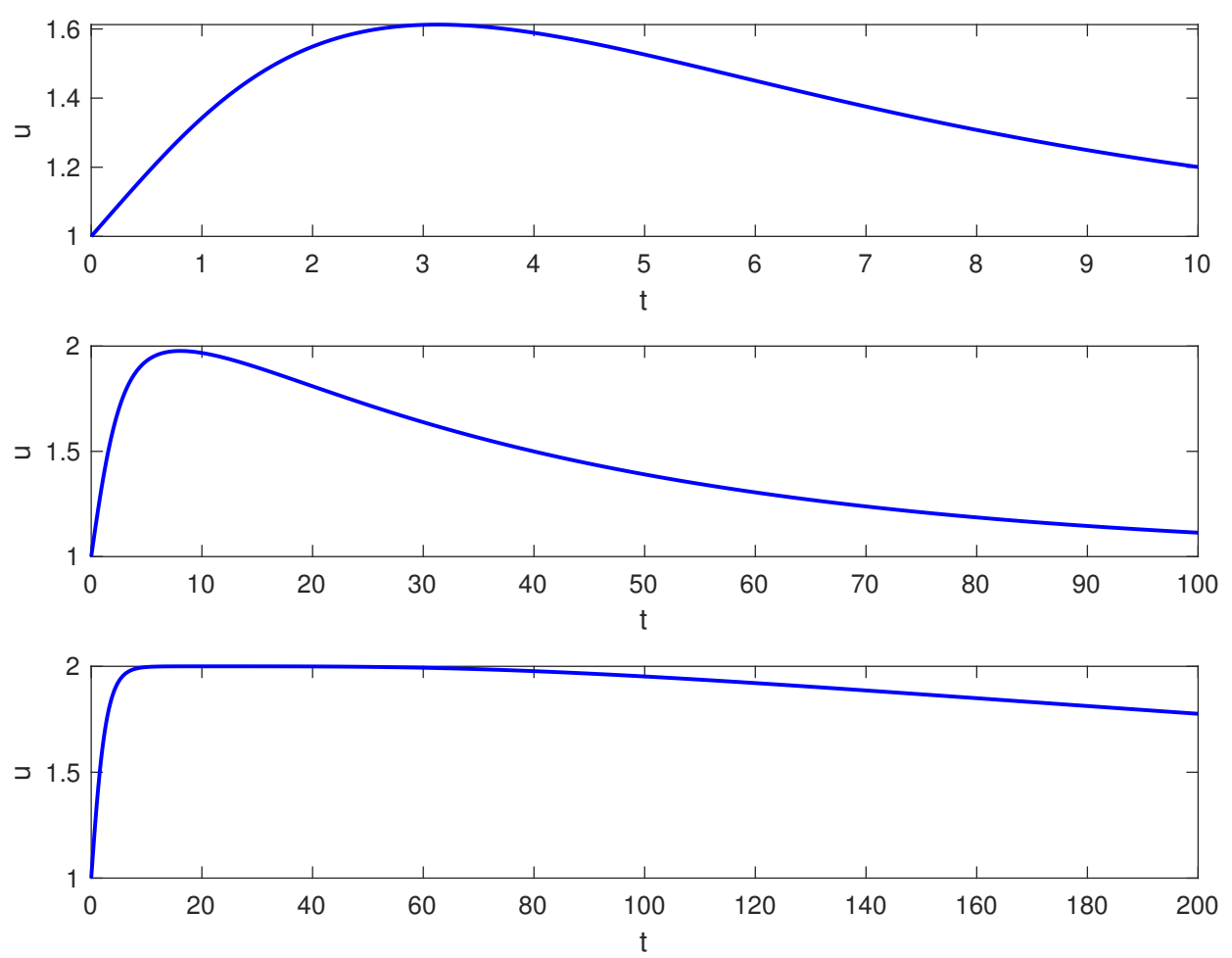

Figure 6 . The numerical results for $u$ at $x=0$ satisfying equations 2.2 2.3 with very mild parameter values $E=R=A=\rho=\Omega=c_{v}=B=C=1$ and $\kappa=0.1,0.01,0.001$ (top to bottom). Overshoot is clearly seen for all cases here and lasts longest for the lowest $\kappa$ value.

Wall layers are necessary partly because the core equations have no spatial dependence and represent in effect only initial value problems. As a result, the loss of the derivative term in $x$ means that the underlying boundary conditions in 2.7 a cannot be satisfied in the core, in the general case. We seek an accompanying solution to the core solutions by considering the system (2.6) near the boundaries. To handle the extreme parameter $\bar{\kappa}$ we set, in the left-hand thin wall layer,

$$
x=-1+\bar{\kappa}^{1 / 2} \bar{x}
$$

and expand

$$
\begin{aligned}
\bar{u} & =\bar{u}_{e}(\bar{x}, \bar{t})+\ldots, \\
\alpha & =\alpha_{e}(\bar{x}, \bar{t})+\ldots
\end{aligned}
$$



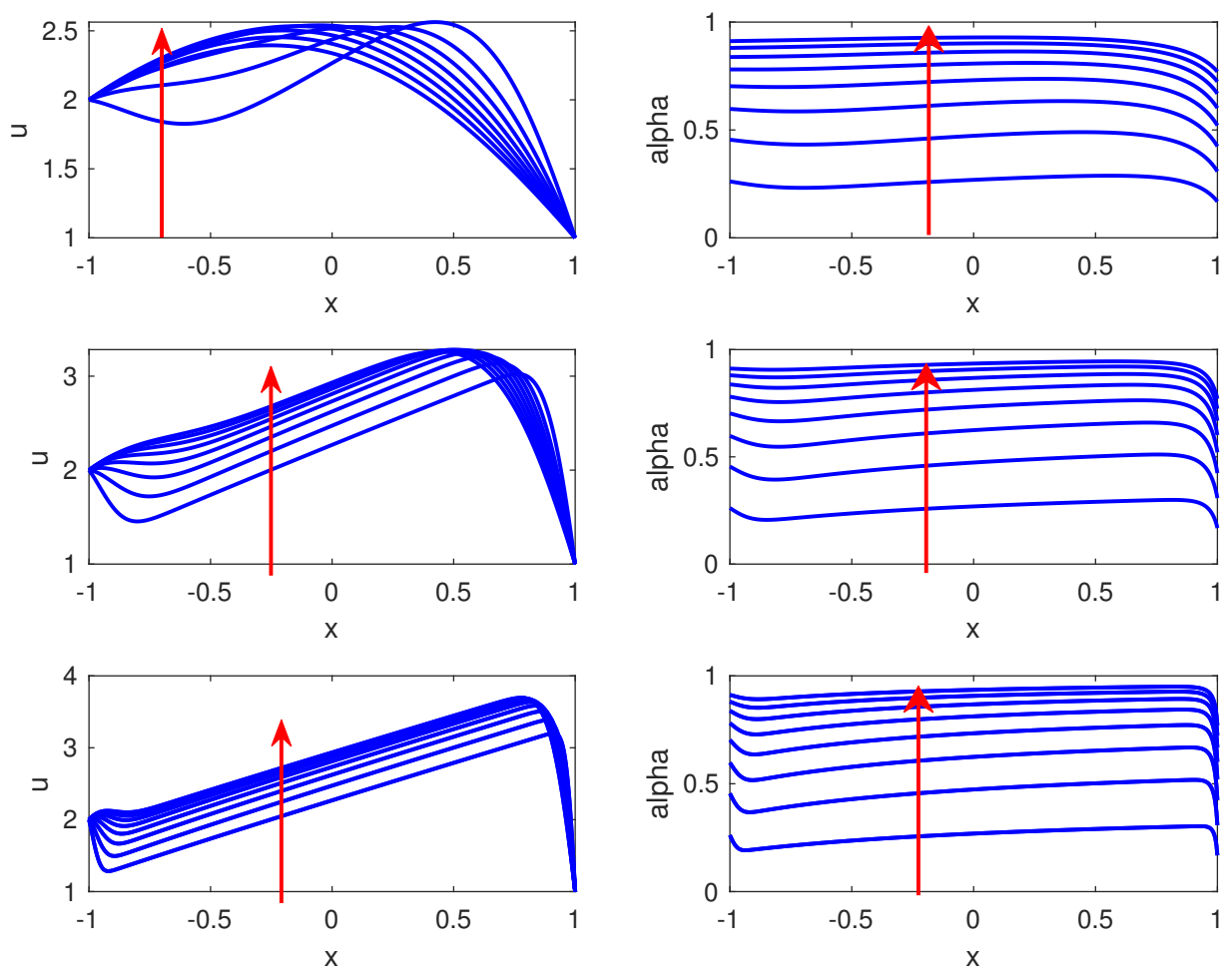

Figure 7. The numerical results for $u, \alpha$ for the non-symmetric case satisfying equations (2.2) - 2.3 with very mild parameter values $E=R=A=\rho=\Omega=c_{v}=1$ and $\kappa=$ $0.1,0.01,0.001$ (top to bottom). The temperature $u$, with boundary conditions $u(-1, t)=$ 2 and $u(1, t)=1$, is shown for fixed values of $t=0$ to $t=4$ in steps of $1 / 2$, where the red vertical arrows indicate time increasing. Here the initial temperature is given by $C(x)=x+2$.

Substitution into 2.6 implies that the governing equations are

$$
\begin{aligned}
\frac{\partial \overline{u_{e}}}{\partial \bar{t}} & =\frac{\partial^{2} \bar{u}_{e}}{\partial \bar{x}^{2}}+\bar{\Omega} \frac{\partial \alpha_{e}}{\partial \bar{t}}, \\
\frac{\partial \alpha_{e}}{\partial \bar{t}} & =\left(1-\alpha_{e}\right) \exp \left(-\frac{1}{\bar{u}_{e}}\right),
\end{aligned}
$$

in the wall layer. (For the most realistic cases described earlier the edge layer is remarkably thin, having an approximate non-dimensional thickness of order $10^{-12}$ from (3.6). The validity of the continuum model may become questionable there for such cases. Further discussion on this is presented in 6,

It is notable that since at present the core problem is independent of $x$, the solutions at the inside edges of the wall layers are valid across the entire core. In other words, the solutions $\bar{u}_{e}$ and $\alpha_{e}$ satisfying (3.8) coincide with the core solutions at $\bar{x}=\infty$ effectively. 

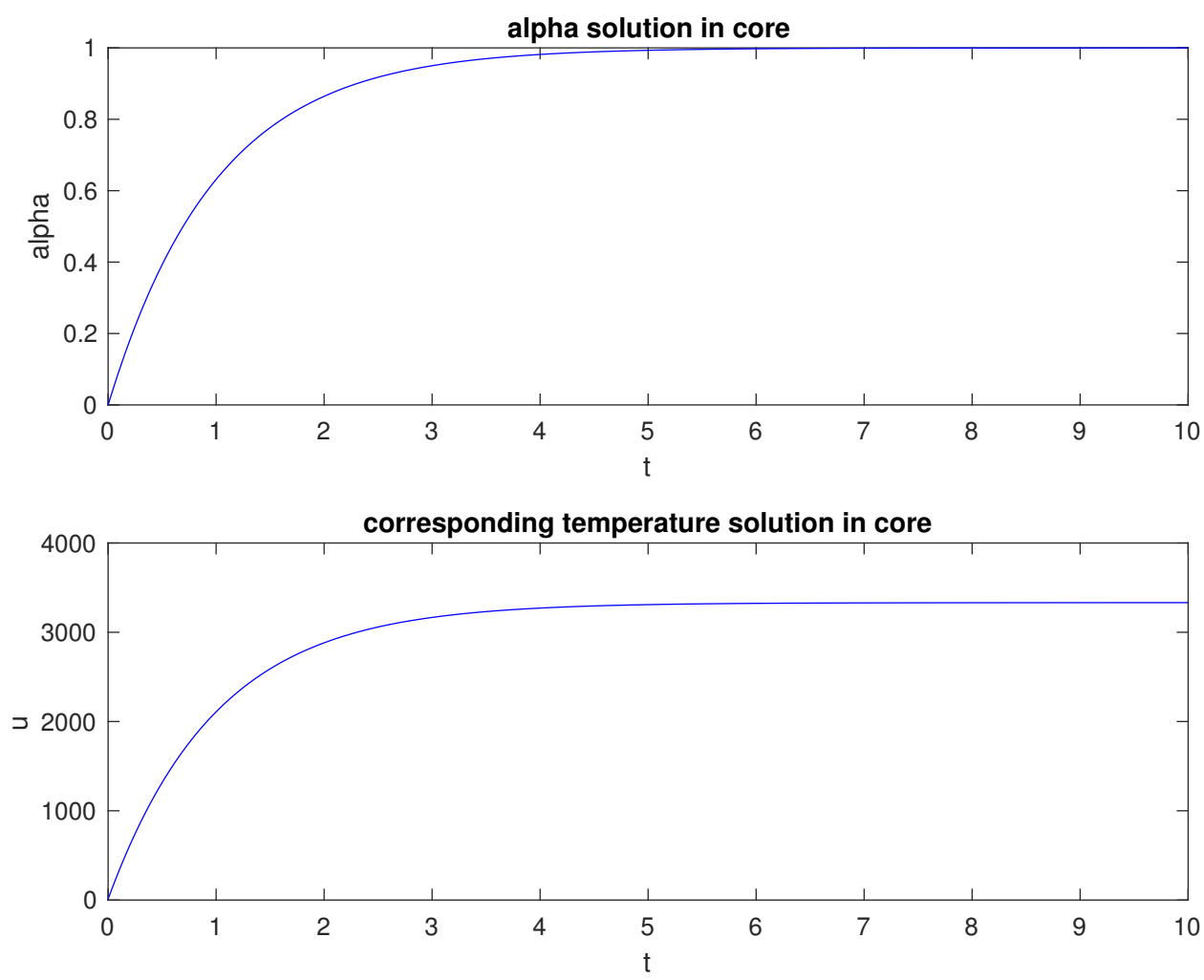

Figure 8. The solutions $\alpha$ (top) and $u$ (bottom) in the core satisfying equation (3.5).

We therefore subject (3.8) to the boundary conditions

$$
\bar{u}_{e}(\infty, \bar{t})=\bar{u}_{c}, \quad \alpha_{e}(\infty, \bar{t})=\alpha_{c} .
$$

It also holds that

$$
\bar{u}_{e}(0, \bar{t})=\bar{B},
$$

and, as before, the boundary solution for $\alpha_{e}$ at the wall can be determined by solving the second equation in (3.8) using the condition (3.9). That

$$
\alpha_{e}(0, \bar{t})=1-\exp \left(-\bar{t} \exp \left(-\frac{E}{R B}\right)\right),
$$

at the wall. We also have the initial conditions

$$
\bar{u}_{e}(\bar{x}, 0)=\bar{C}, \quad \alpha_{e}(\bar{x}, 0)=0, \quad 0<\bar{x}<\infty .
$$

Again we note that there is a similarity solution for small times $\bar{t}$ near $\bar{x}=0,31$. To solve the system 3.8 we use the finite difference schemes of Section 2 . Typically we took $0 \leqslant \bar{x} \leqslant 1000$, to ensure the spatial domain was large enough to demonstrate the true solution behaviour. In Figure 10 we plot the numerical solutions to $\bar{u}_{e}$ and $\alpha_{e}$ using 

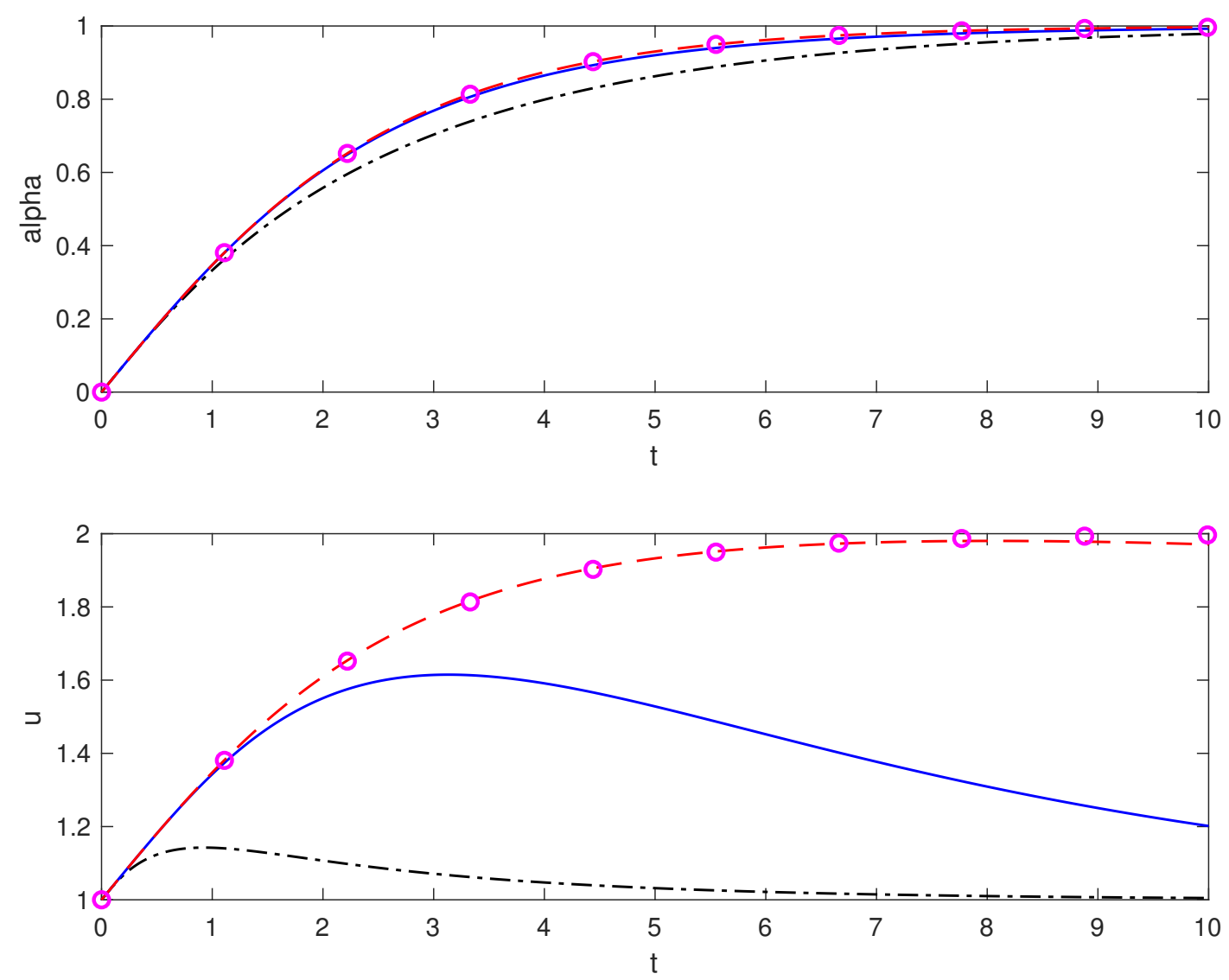

Figure 9. Comparison of core (round markers) and full solutions for varying $\kappa=$ 1, 0.1, 0.01 (represented by the dot-dashed black, solid blue and dashed red lines, respectively).

the mild parameters $E=20 ; A=200 ; C=15$ and $B=45$. In Figure 11 we compare the full non-asymptotic solutions given in Figure 4 (top) with the present asymptotic wall solutions (bottom). Note that to compare these solutions directly we must scale $u$ and $T_{\max }$ in Figure 4 by $\frac{R}{E}$ and $A$ respectively. We observe that the solutions not on the boundary (which is ultimately what we are interested in, since we do not know them a priori) match almost perfectly.

At sufficiently large times $\bar{t}$ the wall layer solution acquires a similarity form. This is because the core temperature and $\alpha_{e}$ both asymptote to constants then, in particular with the core temperature being $\bar{C}+\bar{\Omega}$ and $\alpha_{e}$ being unity to a first approximation, and on the other hand the sidewall temperature $\bar{B}$ is taken to be constant. So in the wall layer $\bar{u}_{e}$ is expected to be of $\mathcal{O}(1)$ and $\alpha_{e}$ is anticipated as being unity with only an exponentially small correction in view of $(3.8 \mathrm{~b})$. The orders of magnitude involved then 


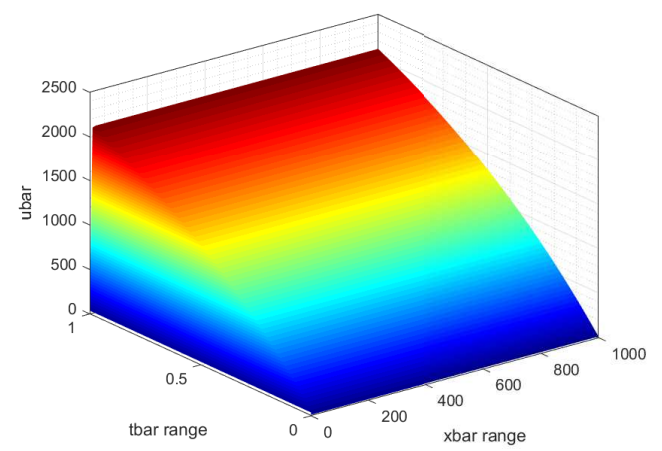

(a) $\bar{u}_{e}$

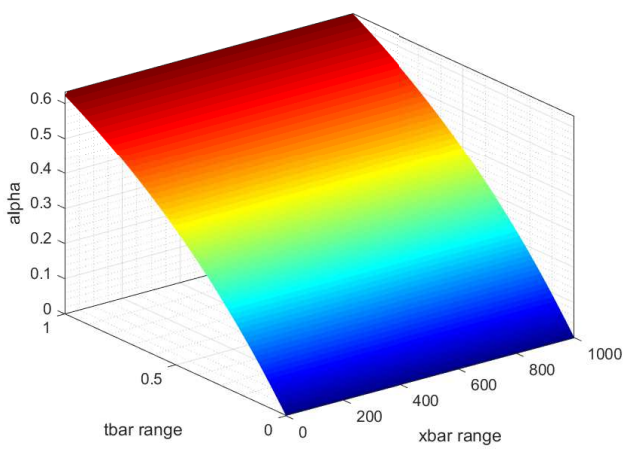

(b) $\alpha_{e}$

Figure 10. The wall-layer solutions $\bar{u}_{e}$ and $\alpha_{e}$ satisfying 3.8 using a second-order numerical scheme with the mild parameters $E=20 ; A=200 ; C=15$ and $B=45$. Note that the $\bar{x}$ range has been truncated to facilitate a numerical implementation.
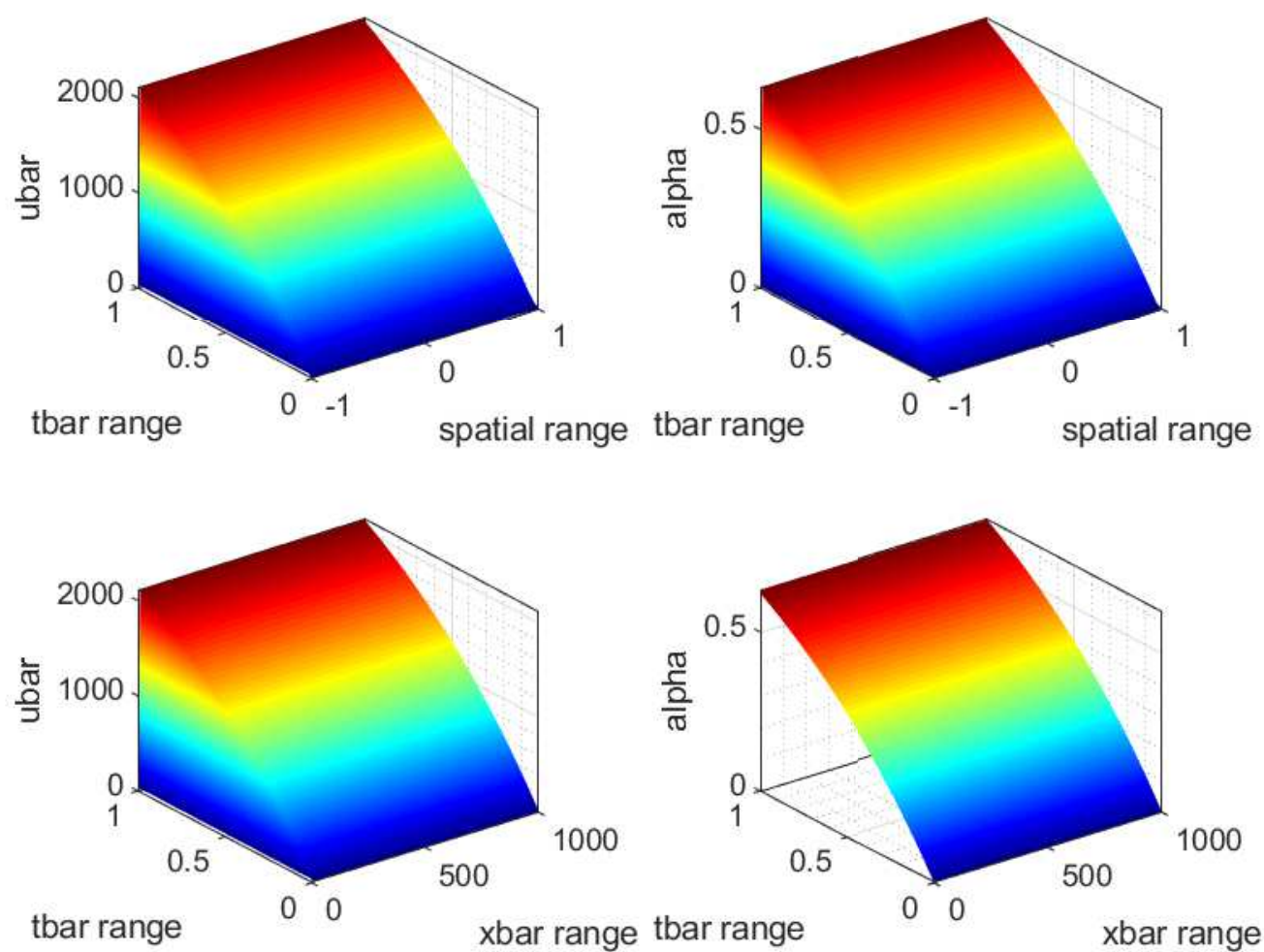

Figure 11. Comparison of solutions $u$ and $\alpha$ for the full system (top) corresponding to equations (2.2), (2.3) and the wall-layer (bottom) solutions corresponding to equation 3.8 . 
suggest that the $\bar{x}$ scale grows as $\bar{t}^{1 / 2}$, leading to the expression

$$
\bar{u}_{e}=f_{1}(\xi)+\ldots, \quad \alpha_{e}=1+f_{2}(\xi)+\ldots
$$

where $\xi=\bar{x} / \vec{t}^{1 / 2}$ is of order unity and the function $f_{2}$ is exponentially small. Substituting into (3.8) we obtain the equation $-\frac{1}{2} \xi f_{1}^{\prime}=f_{1}^{\prime \prime}$ for $f_{1}(\xi)$, with no influence retained now from the reaction effects. The equation is a classical thermal one and yields the solution

$$
f_{1}(\xi)=(\bar{C}+\bar{\Omega}-\bar{B}) I^{-1} \int_{0}^{\xi} \exp \left(-\frac{1}{4} \xi^{2}\right) d \xi+\bar{B} .
$$

Here $I=\pi^{1 / 2}$.

The second major time scale arises because on a longer time scale the wall layers penetrate into the core and become one with it. The wall layer thickness increases like $\bar{t}^{1 / 2}$ and so makes its presence felt in the core over a long time scale $\bar{t}$ of order $\bar{\kappa}^{-1}$, from the scalings above, specifically in $(3.7 a)$. The evolution at that stage takes the form

$$
\begin{array}{ll}
\bar{u}=\mathcal{O}(1), & \alpha=1+\epsilon, \\
x=\mathcal{O}(1), & \bar{t}=\bar{\kappa}^{-1} \hat{t}
\end{array}
$$

over the entire domain, where $\epsilon$ denotes an exponentially small term. The governing equation $2.6 \mathrm{a}$ ) thus reduces to the classical thermal one

$$
\frac{\partial \bar{u}}{\partial \hat{t}}=\frac{\partial^{2} \bar{u}}{\partial x^{2}}
$$

while $2.6 \mathrm{~b}$ gives only effects of higher order. The boundary and initial conditions for (3.15) are

$$
\begin{aligned}
& \bar{u}=\bar{B} \quad \text { at } x= \pm 1 \quad \text { for } \hat{t}>0, \\
& \bar{u}=\bar{C}+\bar{\Omega} \quad \text { at } \hat{t}=0^{+} \quad \text { for }|x|<1 .
\end{aligned}
$$

The problem can be transformed to one solved in Carslaw and Jaeger [31], showing similarity behaviour near each wall at early times but a steady state of $\bar{u}$ equal to $\bar{B}$ being approached at late times. The initial (core) temperature can clearly be substantially larger than the steady-state temperature over this time scale. Moreover the complete behaviour of the temperature $\bar{u}$ is seen to be on a different (longer) time scale than the reaction rate $\alpha$.

\subsection{Effects of other parameters $(\bar{\Omega}, \bar{C}, \bar{B})$.}

Although $\bar{\kappa}$ is by far the most extreme parameter the small typical values of the scaled initial temperature $\bar{C}$ and the scaled reaction constant $\bar{\Omega}$ still play important roles in the core of the interaction. Numerical solutions obtained for $(\bar{\Omega}, \bar{C})$ values of $(1,0.1),(0.1,1)$, $(0.1,0.1),(10,1),(1,1)$ are shown in Figure 12 (a)-(e) respectively.

Suppose first that $\bar{C}$ is small and $\bar{\Omega}$ is $\mathcal{O}(1)$. Then in the core equation $(3.5)$ the righthand side, when plotted as a function of $\alpha_{c}$, is exponentially small of order $c=\exp (-1 / \bar{C})$ at zero $\alpha_{c}$ but rises rapidly as $\alpha_{c}$ increases to $\mathcal{O}(\bar{C})$ and it asymptotes towards unity as $\alpha_{c}$ increases further. The influence of the $\left(1-\alpha_{c}\right)$ term then gradually reduces the right-hand side over a slower scale. The function $d \bar{t} / d \alpha_{c}$ therefore decreases rapidly from 
its initial exponentially large value at zero $\alpha_{c}$ to unity when $\alpha_{c}$ becomes larger than $\mathcal{O}(\bar{C})$ but still small. Clearly $\alpha_{c}$ as a function of time begins small, of order $\bar{C}$, and remains so for a considerable time. This slow effect on the reaction when the initial temperature is small makes sense physically. The main evolution of $\alpha_{c}$ into an $\mathcal{O}(1)$ quantity occurs when time $\bar{t}$ is exponentially large,

$$
\bar{t}=\exp \left(\frac{1}{\bar{C}}\right)+\overline{\bar{t}}
$$

with $\overline{\bar{t}}$ being of $\mathcal{O}(1)$ and the governing equation then becoming

$$
\frac{d \alpha_{c}}{d \overline{\bar{t}}}=\left(1-\alpha_{c}\right) \exp \left(-\frac{1}{\bar{\Omega} \alpha_{c}}\right) \text {. }
$$

See Figure 12(a). The march back in time corresponds to $\alpha_{c}$ being small, such that (3.19) gives, after some working,

$$
\alpha_{c} \sim \bar{\Omega}^{-1}(\ln |\overline{\bar{t}}|)^{-1}
$$

which confirms the slow progress of the evolution. The march forward in time is effectively displayed in Figure 12(a), giving rise to $\alpha_{c}$ tending to unity at large $\overline{\bar{t}}$.

Secondly, if on the other hand $\bar{C}$ is of order unity but $\bar{\Omega}$ is small as in Figure 12 (b) then the contribution $\bar{\Omega} \alpha_{c}$ in 3.5 simply plays a negligible part throughout the interaction. The governing equation reduces to a linear ODE for $\alpha_{c}$ to leading order and the form

$$
\alpha_{c}=1-\exp (-c \bar{t})
$$

describes the solution, where $c=\exp (-1 / \bar{C})$ is now a given $\mathcal{O}(1)$ constant in the present case of $\bar{C}$ being uniform. The time scale in terms of $\bar{t}$ thus remains of order unity. The low reaction rate here means physically that the core temperature remains constant to leading order.

Thirdly, if $\bar{C}$ and $\bar{\Omega}$ are both small as in Figure 12 (c) and of order $\Delta$, say $\Delta(\overline{\bar{C}}, \overline{\bar{\Omega}})$ respectively, then again consideration of the graph of the function $d \bar{t} / d \alpha_{c}$ is helpful. Its initial value is large, being $1 / c$, and the function remains large and positive through the entire interaction, reaching an exponentially large minimum value of

$$
\overline{\bar{\Omega}} \Delta^{-1}(\overline{\bar{C}}+\overline{\bar{\Omega}})^{-2} \exp \left(\Delta^{-1}(\overline{\bar{C}}+\overline{\bar{\Omega}})^{-1}\right),
$$

at the value

$$
\alpha_{c}=1-\frac{\Delta(\overline{\bar{C}}+\overline{\bar{\Omega}})^{2}}{\overline{\bar{\Omega}}},
$$

i.e. near the end of the reaction. Hence the time $\bar{t}$ taken for the reaction to be completed such that $\alpha_{c}$ grows from zero to unity is exponentially large again. The physical sense is similar to that in the case of (3.18)- $(3.20)$ concerning relatively slow reaction.

By contrast, if either or both of $\bar{C}$ and $\bar{\Omega}$ is or are large then the representative scale $\bar{t}$ remains $\mathcal{O}(1)$, with $\alpha_{c}$ given by $1-\exp (-\bar{t})$ and the scaled temperature $\bar{u}_{c}$ given by

$$
\bar{u}_{c}=\bar{C}+\bar{\Omega}(1-\exp (-\bar{t})) .
$$

An example is shown in Figure 12(d). The maximum temperature $\bar{C}+\bar{\Omega}$ is approached at times $\bar{t}$ which are large but not as large as in the cases of 3.18 - 3.20 and 3.23 . 

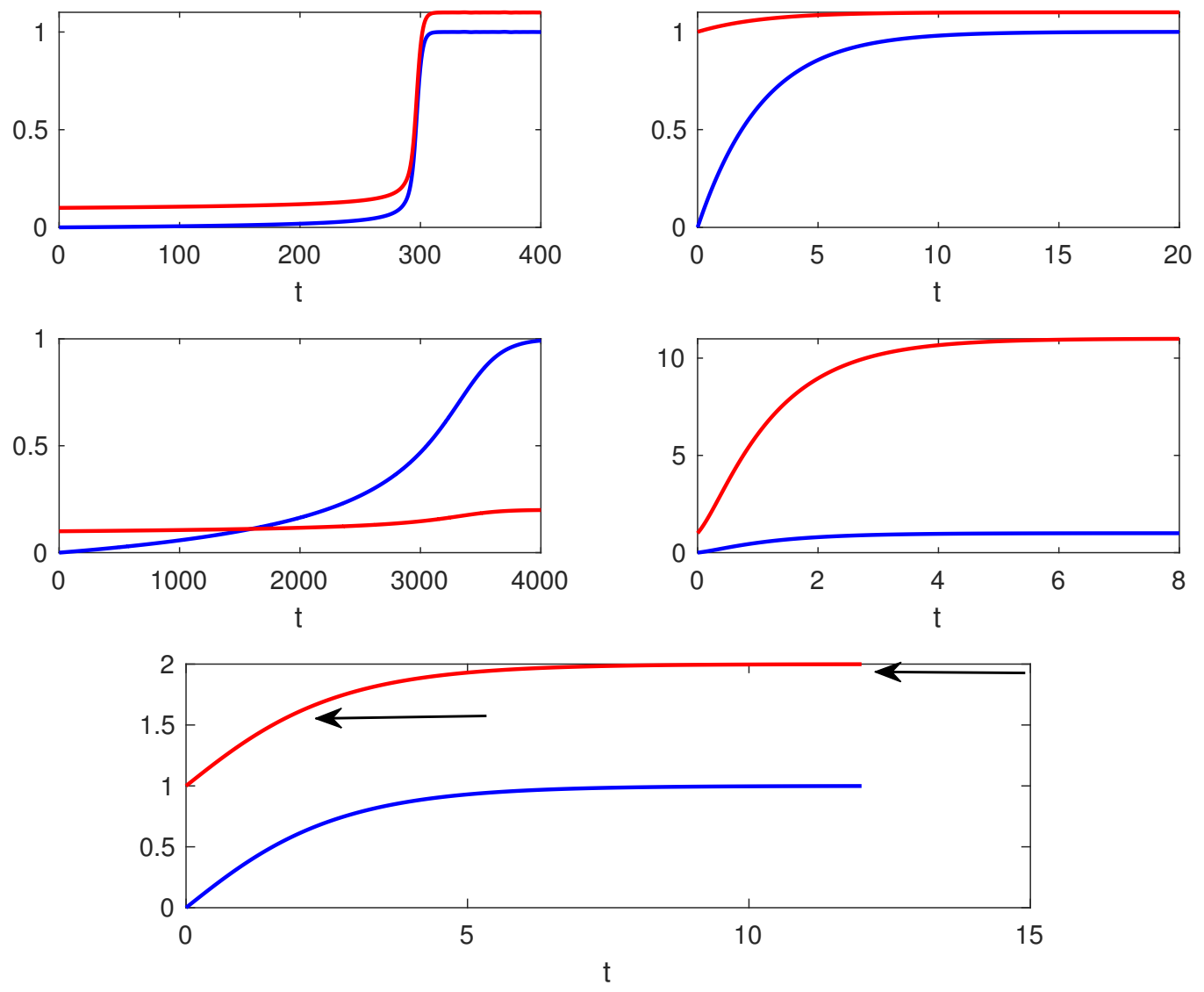

Figure 12. Core solutions $\bar{u}_{c}$ (red) and $\alpha_{c}$ (blue) corresponding to Equation (3.19) as parameters $(\bar{\Omega}, \bar{C})=(1,0.1),(0.1,1),(0.1,0.1),(10,1),(1,1)$, (left to right - top to bottom). In the bottom figure, the arrows represents the highest and mid-values of the temperature $\bar{u}_{c}$.

The effect of the sidewall temperature $\bar{B}$ is felt only in the wall layer, at least for times $\bar{t}$ of order unity because the core is unaffected to leading order by diffusion in $x$. This is discussed further now. The case of relatively small $\bar{B}$, corresponding to a relatively low temperature imposed at the wall, is also of some mathematical concern although of little likely relevance to real application. Since any $\bar{u}$ of $\mathcal{O}(1)$ leads to $\alpha_{e}$ tending to unity at large $\bar{t}$ the similarity solution (19)-(20) still holds then for most $\xi$ values but with the inner boundary condition of $\bar{u}\left(=f_{1}\right.$ to leading order) equalling $0+$ at $\xi=0+$ and $\bar{u}$ tending linearly to zero with $\xi$. Hence if $\bar{B}=\epsilon \hat{B}$ say with $\epsilon$ being small and $\hat{B}$ of order unity then there is a sublayer close to the wall where $\xi, \bar{u}$ are both $\mathcal{O}(\epsilon)$ and $\bar{u}$ is affected by the low wall temperature. The second stage where $\hat{t}$ is $\mathcal{O}(1)$ is perhaps clearer to analyse. Here the majority of the domain is governed by (23)-(25) but subject 
to $\bar{u} \rightarrow 0+$ as $x \rightarrow-1+$ and similarly as $x \rightarrow 1-$. So the behaviour

$$
\bar{u} \sim(x+1) \beta(\hat{t}) \quad \text { at } x=-1+
$$

is expected near the left-hand wall where $\beta$ is an order-unity function of $\hat{t}$ determined by the forward-marching solution of (23)-(25). Essentially the same response occurs at the right-hand wall. The function $\beta(\hat{t})$, and indeed the entire $\bar{u}(x, \hat{t})$ solution, tends to zero at large times $\hat{t}$, while at small times $\beta(\hat{t})$ is large, varying as $\hat{t}^{-1 / 2}$. Near the wall on the other hand a sublayer is present in which

$$
\bar{u}=\epsilon \hat{u}+\ldots, \quad \alpha=\hat{\alpha}+\ldots, \quad \text { with } x=-1+\epsilon \hat{x}
$$

where $\hat{\alpha}$ is non-trivial because of the low wall temperature. The governing equations $(2.6 a), 2.6 b$ in this sublayer reduce to

$$
\begin{aligned}
\frac{\partial^{2} \hat{u}}{\partial \hat{x}^{2}} & =0, \\
\bar{\kappa} \frac{\partial \hat{\alpha}}{\partial \hat{t}} & =(1-\hat{\alpha}) \exp \left(-\frac{1}{\epsilon \hat{u}}\right) .
\end{aligned}
$$

Here $3.27 a$ gives, on matching with 3.25,

$$
\hat{u}=\hat{a}(\hat{t})+\hat{x} \beta(\hat{t}),
$$

with $\hat{a}=\hat{B}$ independently of $\hat{t}$ due to the wall condition. The balance in $3.27 b$ which is akin to those operating in $(3.18)-(3.24)$ is associated with a critical size of $\epsilon$ being

$$
\epsilon=-G /(\ln \bar{\kappa})
$$

with $G$ being an $\mathcal{O}(1)$ positive constant and a critical temperature being reached at which

$$
\hat{u}=G^{-1}+\epsilon u_{1}+\ldots
$$

where $(3.27 b)$ becomes at leading order

$$
\frac{\partial \hat{\alpha}}{\partial \hat{t}}=(1-\hat{\alpha}) \exp \left(G^{2} u_{1}(\hat{x}, \hat{t})\right) .
$$

The form 3.31 containing $\mathcal{O}(1)$ quantities ensures, when combined with $3.28,3.30$, that $\hat{\alpha}$ tends to unity as time increases over the present scale.

\subsection{Comparisons}

The asymptotic description in the previous two subsections appears to capture all the major trends of the full solutions as $\kappa$ or $\bar{\kappa}$ is decreased and hence the major trends for the realistic case also in which $\kappa$ and $\bar{\kappa}$ are extremely small. The main quantitative comparisons are shown by means of the arrows displayed in Figure 12 (e) as well as the round markers in Figure 9, for the same case. To clarify, the arrows in Figure 12(e), which indicate the maximum $\alpha$ value reached and the time taken for $\alpha$ to attain the average of its maximum and its initial value, tend to confirm the agreement seen in Figure 9 . See also the comparisons presented in Figure 11. Overall the analysis is seen to 'work' for values of $\bar{\kappa}$ below about 0.01 say and hence for the extremely small $\bar{\kappa}$ values of the realistic setting. 
Further it is notable that the maximum temperature attained can be predicted in analytical form very readily from the core responses: the maximum is

$$
\bar{u}_{\max }=\bar{\Omega}+\bar{C}
$$

in non-dimensional terms. This is because of the result $(3.4)$ in the core when $\alpha_{c}$ tends to unity at the completion of the reaction. In dimensional terms 3.32 yields

$$
u_{c, \max }=3840^{\circ} \mathrm{K}
$$

for the maximum, based on the parameters $(\bar{C}, \bar{\Omega}, E / R)$ being $(0.01,0.15,24000)$ as representative values from Table 1 . The predicted maximum temperature in $(3.32,3.33)$ is independent of the imposed wall temperatures and is of much potential interest.

\section{Computational properties for three reactants}

A multi-kinetic reaction process is now introduced into the model to provide an improved representation of the physical and chemical processes that may occur in practice. See 1 ] for further detail of the model. Following the work of [21], 17] for example we consider here a three-step reaction to model better the endothermic and exothermic chemical processes.

The diffusion process with a three-step reaction present is described by

$$
\begin{aligned}
& \rho c_{v} \frac{\partial u}{\partial t}=\kappa \frac{\partial^{2} u}{\partial x^{2}} \\
& \quad+N_{A} Q_{1} Z_{1} \exp \left(-\frac{E_{1}}{R u}\right)+N_{B} Q_{2} Z_{2} \exp \left(-\frac{E_{2}}{R u}\right)+N_{C}^{2} Q_{3} Z_{3} \exp \left(-\frac{E_{3}}{R u}\right) .
\end{aligned}
$$

Coupled with 4.1) are the following ODEs governing the rates of change of the reactions $N_{A}, N_{B}, N_{C}$ respectively,

$$
\begin{aligned}
& \dot{N}_{A}=-\frac{N_{A} Z_{1}}{\rho} \exp \left(-\frac{E_{1}}{R u}\right), \\
& \dot{N}_{B}=\frac{N_{A} Z_{1}}{\rho} \exp \left(-\frac{E_{1}}{R u}\right)-\frac{N_{B} Z_{2}}{\rho} \exp \left(-\frac{E_{2}}{R u}\right), \\
& \dot{N}_{C}=\frac{N_{B} Z_{2}}{\rho} \exp \left(-\frac{E_{2}}{R u}\right)-\frac{N_{C}^{2} Z_{3}}{\rho} \exp \left(-\frac{E_{3}}{R u}\right),
\end{aligned}
$$

and

$$
N_{A}+N_{B}+N_{C}+N_{D}=1
$$

Here the dots denote derivatives with respect to time. The constants $Q_{1}-Q_{3}$ in the reactant equations stand for the heats of reaction whereas $E_{1}-E_{3}$ are the corresponding activation energy constants of the reactions and $Z_{1}-Z_{3}$ are the corresponding pre-exponential constants. Table 2 presents a list of realistic values for the parameters here.

The boundary and initial conditions for $u(x, t)$ are set as in $2.4 a)$, while the initial conditions on the reactants are

$$
\left(N_{A}, N_{B}, N_{C}\right)=(1,0,0) \text { at } t=0 .
$$

That leaves our task then as solving (4.1)-4.4 subject to $2.4 a$, 4.6. No boundary 


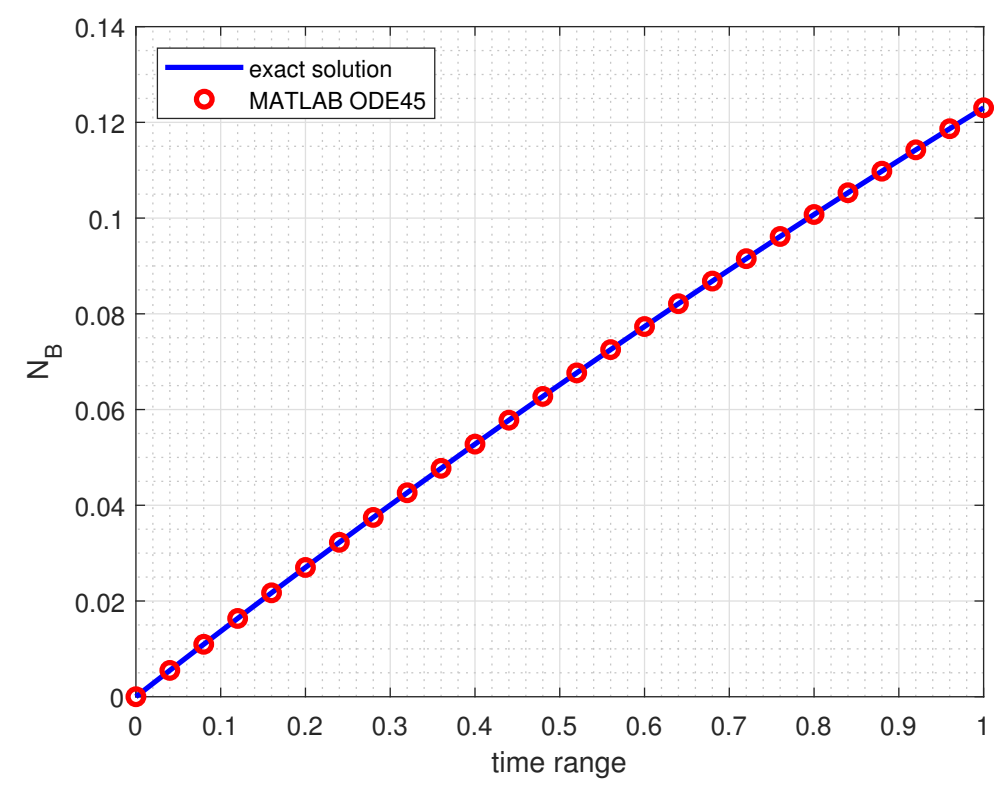

Figure 13. The numerical approximation (using ODE45 in MATLAB) compared with the analytical solution of Equation 4.3

conditions need to be set on the reactant quantities because of the absence of spatial derivatives in the balances (4.2)-(4.4). The value of $N_{A}$ at the boundary where $u=u_{A}$ can be obtained directly from integration of (4.2) and application of (4.6) as

$$
N_{A}=\exp \left(-\frac{Z_{1}}{\rho} \frac{t}{\exp \left(\frac{E_{1}}{R u_{A}}\right)}\right) \text {. }
$$

Hence $N_{B}$ is given by solving (4.3) combined with (4.7) to yield

$$
N_{B}=\frac{A \exp \left(-A \frac{t}{\exp (C)}+D\right)}{B \exp (C)-A \exp (D)}-\frac{A \exp (D)}{B \exp (C)-A \exp (D)} \exp \left(-B \frac{t}{\exp (D)}\right),
$$

where $A=\frac{Z_{1}}{\rho}, B=\frac{Z_{2}}{\rho}, C=\frac{E_{1}}{R u_{A}}$ and $D=\frac{E_{2}}{R u_{A}}$. This is shown graphically in Figure 13 . while $N_{C}$ then stems from (4.4) with 4.7), 4.8), giving the numerical solution presented in Figure 14. These two figures are for the relatively mild parameter values shown in Table 3 together with the boundary condition $u_{A}=45$ which again is comparatively mild.

The computational approach of Section 2 was extended and adapted for the present task. Second-order accuracy in time and space and appropriate lagging were notable features again. Results are plotted in Figure 15. More realistic cases will be re-addressed in Section 6 after the discussion of analytical properties in the following section. 


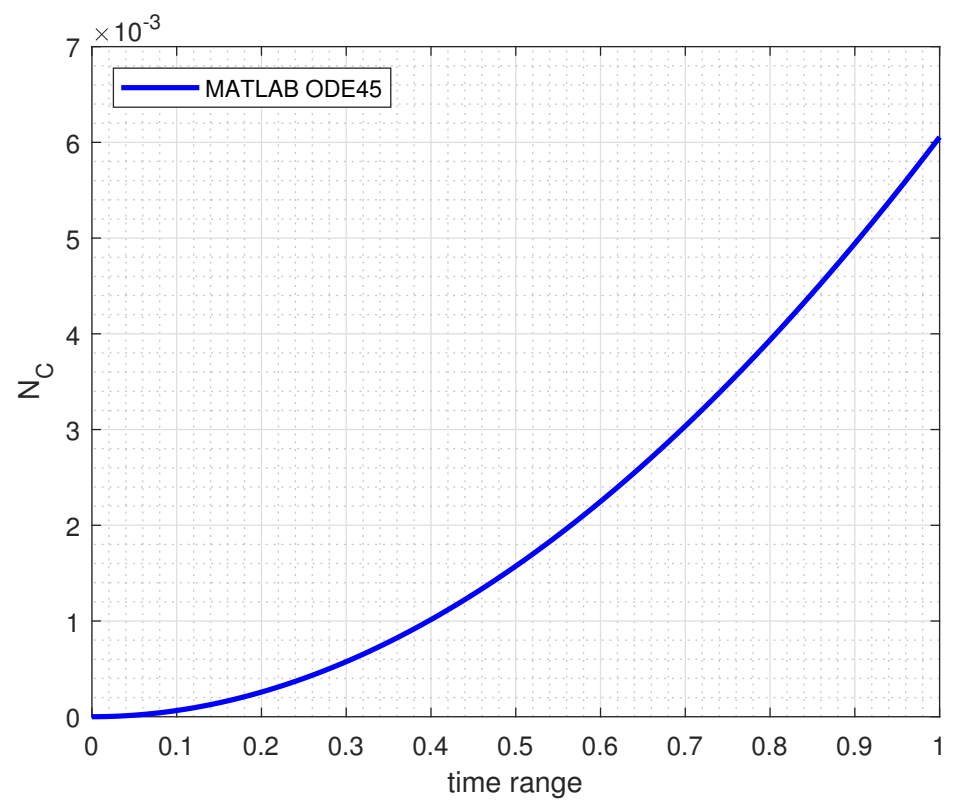

Figure 14. The numerical approximation (using ODE45 in MATLAB) of Equation 4.4

Table 2. Additional parameters used in ODTX model for HMX explosive assuming multistep kinetics as in 21, converted into SI units.

\begin{tabular}{lcc}
\hline \hline Parameter with units & Symbol & Value \\
\hline Heat of first reaction $(\mathrm{J} / \mathrm{kg})$ & $Q_{1}$ & $-4.2 e 5$ \\
Heat of second reaction $(\mathrm{J} / \mathrm{kg})$ & $Q_{2}$ & $1.26 e 6$ \\
Heat of third reaction $(\mathrm{J} / \mathrm{kg})$ & $Q_{3}$ & $5.04 e 6$ \\
Activation Energy of first reaction $(\mathrm{J} / \mathrm{mol})$ & $E_{1}$ & $2.21 e 5$ \\
Activation Energy of second reaction $(\mathrm{J} / \mathrm{mol})$ & $E_{2}$ & $1.85 e 5$ \\
Activation Energy of third reaction $(\mathrm{J} / \mathrm{mol})$ & $E_{3}$ & $1.43 e 5$ \\
First Pre-exponential Constant $\left(\mathrm{kg} \mathrm{m}^{-3} \mathrm{~s}^{-1}\right)$ & $Z_{1}$ & $1.4 e 24$ \\
Second Pre-exponential Constant $\left(\mathrm{kg} \mathrm{m}^{-3} \mathrm{~s}^{-1}\right)$ & $Z_{2}$ & $1.9 e 19$ \\
Third Pre-exponential Constant $\left(\mathrm{kg} \mathrm{m}^{-3} \mathrm{~s}^{-1}\right)$ & $Z_{3}$ & $1.5 e 15$ \\
\hline \hline
\end{tabular}

\section{Analytical solutions and comparisons for three reactants}

Turning to asymptotic analysis for the multi-kinetics interactions we observe that in effect $Q_{n}, Z_{n}$ terms here replace the $\rho \Omega$ and $A$ terms in the one reactant case in Section 2 and the $N$ 's here correspond to the reactant $\alpha$ in the single reactant case. We use the same scalings as before in Section 3 that is we set $t=A^{-1} \bar{t}$ and $u=\frac{E}{R} \bar{u}$ and expand the solution. In addition, to account for the new parameters $Z_{n}=A \bar{Z}_{n} \rho$ and $E_{n}=E \bar{E}_{n}$, we choose $E_{2}$ as a representative value of $E$ to try to ensure $\bar{Z}_{n} \sim \mathcal{O}(1), \bar{E}_{n} \sim \mathcal{O}(1)$, $N_{n} \sim \mathcal{O}(1)$ and $x \sim \mathcal{O}(1)$ to begin. We have again used mild values of $E_{n}, Z_{n}$ and $A$ in the first instance, see Table 3 for exact values. With the scalings above, the controlling 

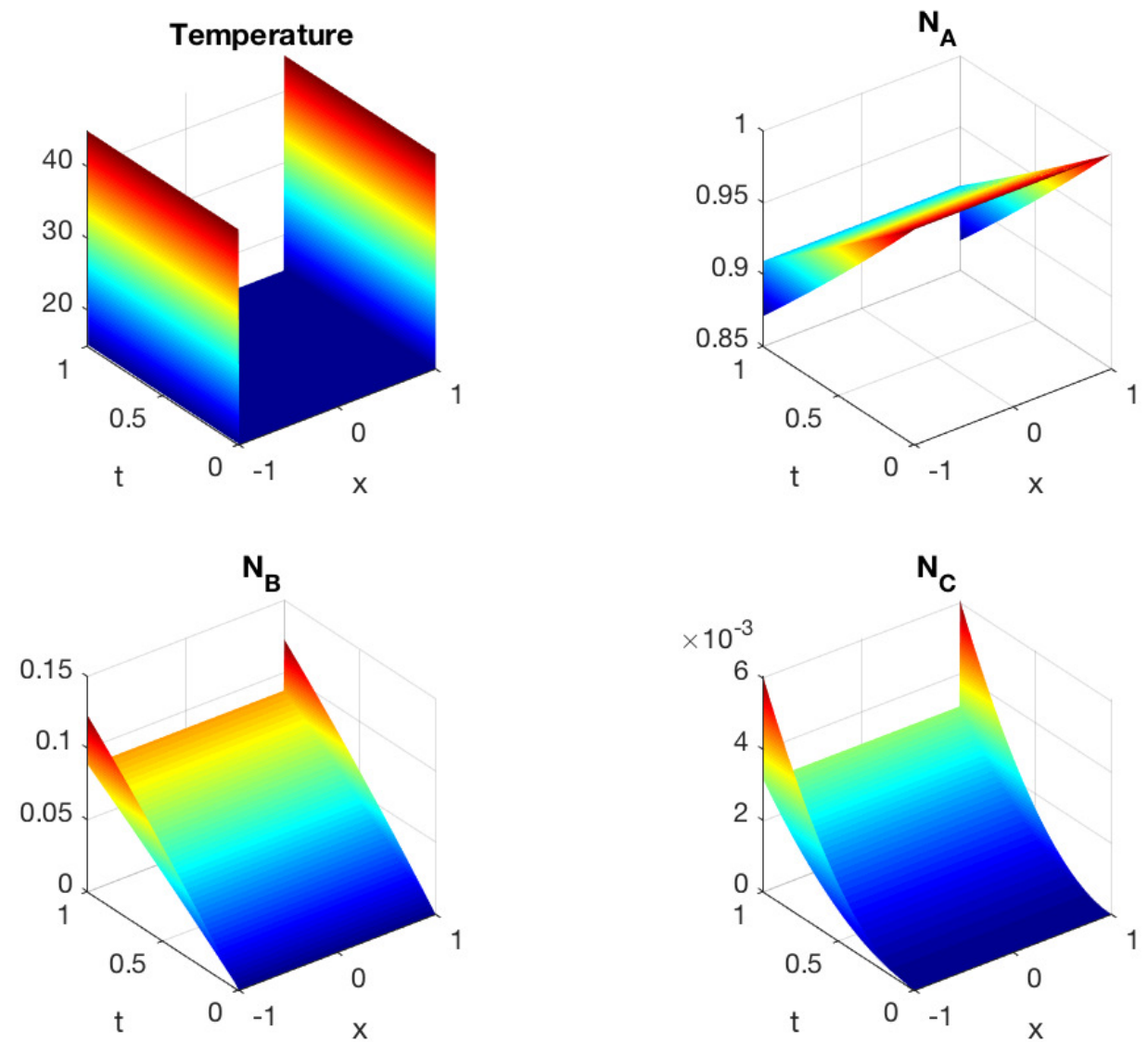

Figure 15. The numerical solutions $\bar{u}, N_{A}, N_{B}$ and $N_{C}$ satisfying 4.1-4.4), using a second-order numerical scheme, with the relatively mild parameters supplied in Table 3.

Table 3. Mild parameters used in analysis of sections 45

\begin{tabular}{lcl}
\hline \hline Parameter with units & Symbol & Value \\
\hline Heat of first reaction $(\mathrm{J} / \mathrm{kg})$ & $Q_{1}$ & -10 \\
Heat of second reaction $(\mathrm{J} / \mathrm{kg})$ & $Q_{2}$ & 20 \\
Heat of third reaction $(\mathrm{J} / \mathrm{kg})$ & $Q_{3}$ & 30 \\
Activation Energy of first reaction $(\mathrm{J} / \mathrm{mol})$ & $E_{1}$ & 70 \\
Activation Energy of second reaction $(\mathrm{J} / \mathrm{mol})$ & $E_{2}$ & 60 \\
Activation Energy of third reaction $(\mathrm{J} / \mathrm{mol})$ & $E_{3}$ & 50 \\
First Pre-exponential Constant $\left(\mathrm{kg} \mathrm{m}^{-3} \mathrm{~s}^{-1}\right)$ & $Z_{1}$ & 300 \\
Second Pre-exponential Constant $\left(\mathrm{kg} \mathrm{m}^{-3} \mathrm{~s}^{-1}\right)$ & $Z_{2}$ & 200 \\
Third Pre-exponential Constant $\left(\mathrm{kg} \mathrm{m}^{-3} \mathrm{~s}^{-1}\right)$ & $Z_{3}$ & 100 \\
Pre-exponential Constant $\left(\mathrm{s}^{-1}\right)$ & $A$ & 200 \\
\hline \hline
\end{tabular}


equations become

$$
\begin{aligned}
\frac{\partial \bar{u}}{\partial \bar{t}} & =\bar{\kappa} \frac{\partial^{2} \bar{u}}{\partial x^{2}}+N_{A} \bar{\Omega}_{1} \exp \left(-\frac{\bar{E}_{1}}{\bar{u}}\right)+N_{B} \bar{\Omega}_{2} \exp \left(-\frac{\bar{E}_{2}}{\bar{u}}\right)+N_{C}^{2} \bar{\Omega}_{3} \exp \left(-\frac{\bar{E}_{3}}{\bar{u}}\right), \\
\frac{d N_{A}}{d \bar{t}} & =-N_{A} \bar{Z}_{1} \exp \left(-\frac{\bar{E}_{1}}{\bar{u}}\right) \\
\frac{d N_{B}}{d \bar{t}} & =N_{A} \bar{Z}_{1} \exp \left(-\frac{\bar{E}_{1}}{\bar{u}}\right)-N_{B} \bar{Z}_{2} \exp \left(-\frac{\bar{E}_{2}}{\bar{u}}\right) \\
\frac{d N_{C}}{d \bar{t}} & =N_{B} \bar{Z}_{2} \exp \left(-\frac{\bar{E}_{2}}{\bar{u}}\right)-N_{C}^{2} \bar{Z}_{3} \exp \left(-\frac{\bar{E}_{3}}{\bar{u}}\right)
\end{aligned}
$$

where $\bar{\kappa}=\frac{\kappa}{\rho c_{v} A}$ which when using the full parameter values gives us $\bar{\kappa} \approx 3.8863 e-27$ and $\bar{\Omega}_{n}=Q_{n} \bar{Z}_{n}\left(\frac{R}{c_{v} E_{2}}\right)$, for $n=1,2,3$. When using relatively mild parameter values we have instead $A=200, \bar{\kappa}=9.7388 e-10$. Thus $\bar{\kappa}$ is the most extreme parameter in both cases, using the full parameter values and milder values. Hence we base our asymptotic analysis on $\bar{\kappa}<<1$ as before in Section 3 . We proceed by again separating the problem into a core and wall layers with equations describing the behaviour in each.

The core problem is reduced for small $\bar{\kappa}$ to Equation 5.1 with a negligible $\frac{\partial^{2} \bar{u}}{\partial x^{2}}$ term, as before in Section 3. Hence, it follows that the quasi-ODE

$$
\frac{d \bar{u}}{d \bar{t}}=N_{A} \bar{\Omega}_{1} \exp \left(-\frac{\bar{E}_{1}}{\bar{u}}\right)+N_{B} \bar{\Omega}_{2} \exp \left(-\frac{\bar{E}_{2}}{\bar{u}}\right)+N_{C}^{2} \bar{\Omega}_{3} \exp \left(-\frac{\bar{E}_{3}}{\bar{u}}\right),
$$

holds in the core, and we have the following ODEs for the reactants $N_{A}, N_{B}$ and $N_{C}$,

$$
\begin{aligned}
& \frac{d N_{A}}{d \bar{t}}=-N_{A} \bar{Z}_{1} \exp \left(-\frac{\bar{E}_{1}}{\bar{u}}\right), \\
& \frac{d N_{B}}{d \bar{t}}=N_{A} \bar{Z}_{1} \exp \left(-\frac{\bar{E}_{1}}{\bar{u}}\right)-N_{B} \bar{Z}_{2} \exp \left(-\frac{\bar{E}_{2}}{\bar{u}}\right), \\
& \frac{d N_{C}}{d \bar{t}}=N_{B} \bar{Z}_{2} \exp \left(-\frac{\bar{E}_{2}}{\bar{u}}\right)-N_{C}^{2} \bar{Z}_{3} \exp \left(-\frac{\bar{E}_{3}}{\bar{u}}\right) .
\end{aligned}
$$

Equations 5.5 - 5.8) can readily be solved numerically subject to the following initial conditions at $\bar{t}=0$,

$$
\begin{aligned}
N_{A}(x, 0) & =1, \\
N_{B}(x, 0) & =N_{C}(x, 0)=0, \\
\bar{u}(x, 0) & =\bar{C}, \quad \bar{C}=\frac{R C}{E_{2}}
\end{aligned}
$$

In Figure 16, the ODEs (5.5)-(5.8) have been solved using the relatively mild parameter values in Table 2 .

In the wall layers the same scaling as that in Section 3 applies, so that $x=-1+\bar{\kappa}^{1 / 2} \bar{x}$ at the left-hand wall and similarly at the right-hand wall. This reduces equation 5.1 to the form

$$
\frac{\partial \bar{u}}{\partial \bar{t}}=\frac{\partial^{2} \bar{u}}{\partial x^{2}}+N_{A} \bar{\Omega}_{1} \exp \left(-\frac{\bar{E}_{1}}{\bar{u}}\right)+N_{B} \bar{\Omega}_{2} \exp \left(-\frac{\bar{E}_{2}}{\bar{u}}\right)+N_{C}^{2} \overline{\Omega_{3}} \exp \left(-\frac{\bar{E}_{3}}{\bar{u}}\right)
$$

independently of $\bar{\kappa}$. To solve the reduced system comprising 5.10 with 5.2 - 5.4 computationally we developed a finite difference scheme based closely on those of Sections 

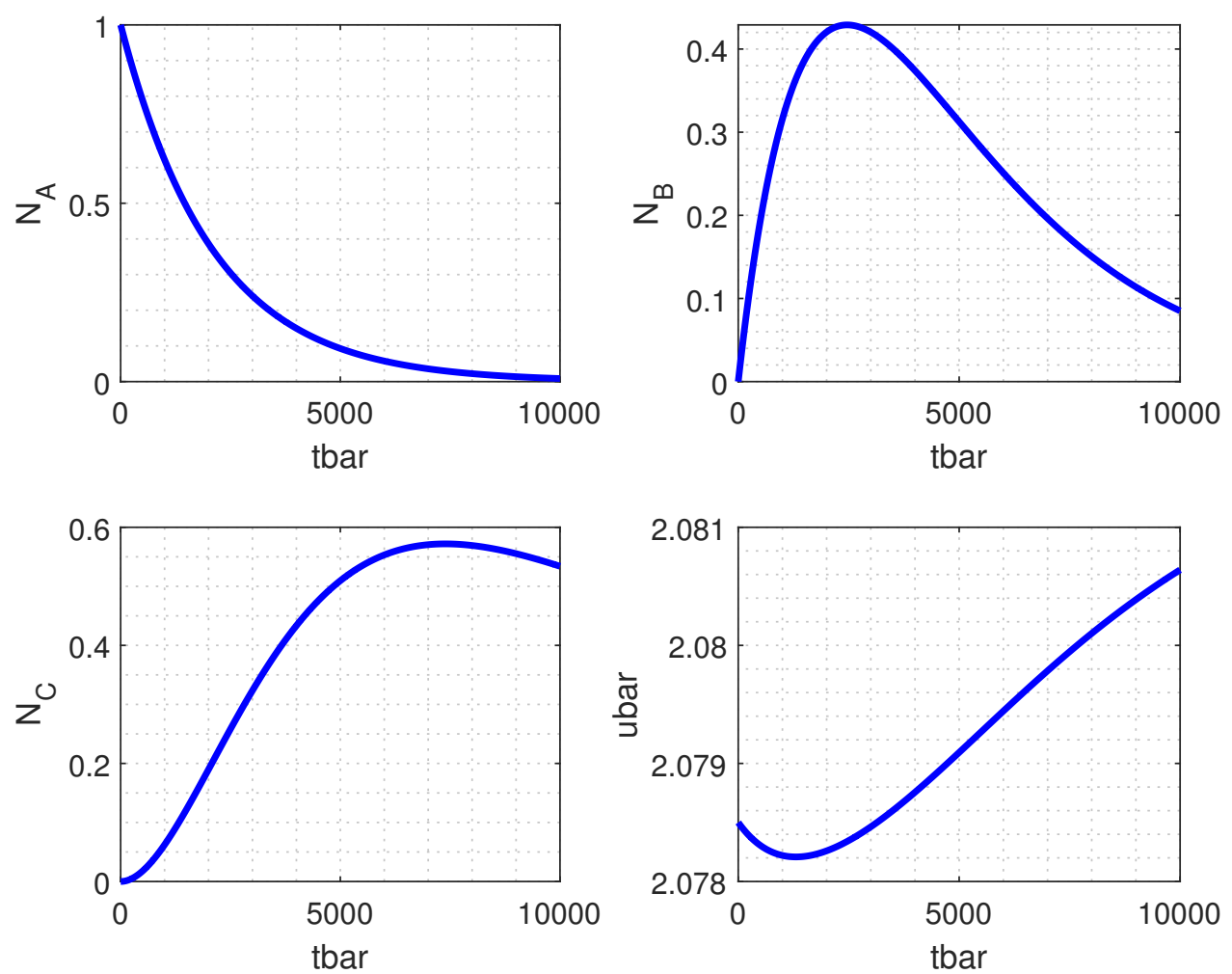

Figure 16. The numerical solutions (using ODE45 in MATLAB) to $\bar{u}, N_{A}, N_{B}$ and $N_{C}$ satisfying Equations 5.5 -5.8. in the core with relatively mild parameter values supplied in Table 3 .

24. Results for the wall layer are presented in Figure 17 where we have used the relatively mild parameter values of Table 3 .

Comparisons given in Figure 18 between the above asymptotic solution and the full numerical solution tend to indicate fair agreement for the milder parameter range of Table 3. These comparisons also indicate significant computational savings using the asymptotic approach.

Further analysis of the core proves to be helpful. Thus (5.5)-(5.8) imply that a certain non-trivial linear combination of the left-hand sides sums to zero and so on integration $\bar{u}$ can be expressed in terms of the reactions in the form

$$
\bar{u}=a_{1}\left(1-N_{A}\right)-a_{2} N_{B}-a_{3} N_{C}+\bar{C} .
$$

Here the initial conditions in (5.9), which include $\bar{C}$, determine the constant of integration and the constant coefficients are given by

$$
a_{n}=\sum_{(n, 3)} \frac{\bar{\Omega}_{n}}{\bar{Z}_{n}}
$$



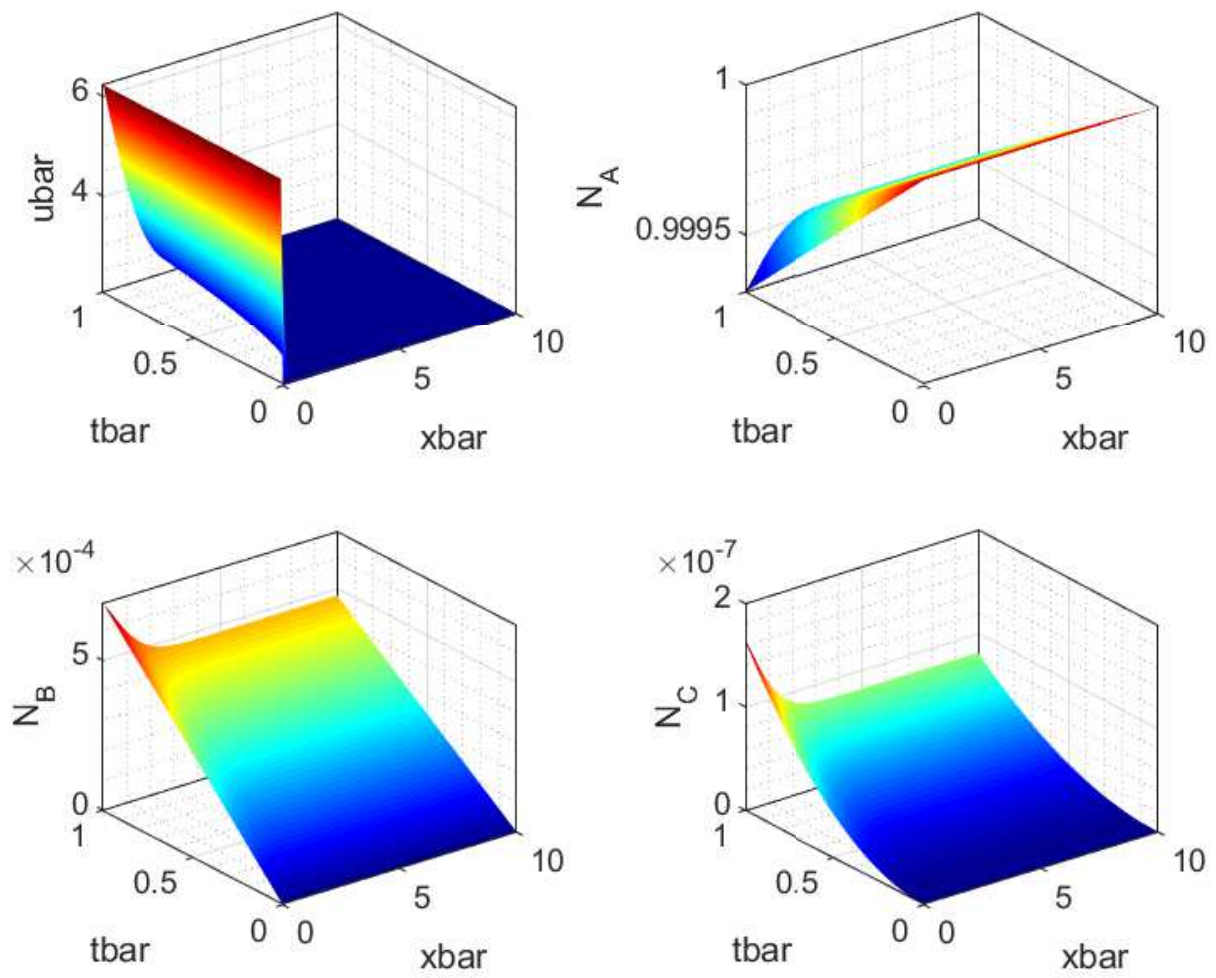

Figure 17. The wall solutions $\bar{u}, N_{A}, N_{B}$ and $N_{C}$ satisfying 5.10 , with the mild parameters supplied in Table 3 . Note that the $\bar{x}$ range has been truncated to facilitate a numerical implementation.

for $n=1,2,3$. We are left with four equations (5.6)-5.8, 5.11) for $N_{A}, N_{B}, N_{C}, \bar{u}$. Sample solutions of this nonlinear system presented in Figure 19 show $\left(N_{A}, N_{B}, N_{C}\right)$ starting as $(1,0,0)$ in line with 5.9 and eventually tending to $(0,0,0)$ at sufficiently large times, with $\bar{u}$ increasing monotonically along with other interesting behaviour during the evolution.

A phase-plane view is also useful here. It follows from treating $N_{B}, N_{C}$ as functions of $N_{A}$ by virtue of eliminating $\bar{t}$ explicitly from (5.6)-(5.8) through division to yield the two equations

$$
\begin{aligned}
& \frac{d N_{B}}{d N_{A}}=-1+\bar{Z}_{21} N_{B} N_{A}^{-1} \exp \left(\frac{\bar{E}_{12}}{\bar{u}}\right) \\
& \frac{d N_{C}}{d N_{A}}=-\bar{Z}_{21} N_{B} N_{A}^{-1} \exp \left(\frac{\bar{E}_{12}}{\bar{u}}\right)+\bar{Z}_{31} N_{C}^{2} N_{A}^{-1} \exp \left(\frac{\bar{E}_{13}}{\bar{u}}\right)
\end{aligned}
$$

with constants $\bar{Z}_{21}=\bar{Z}_{2} / \bar{Z}_{1}, \bar{Z}_{31}=\bar{Z}_{3} / \bar{Z}_{1}, \bar{E}_{12}=\bar{E}_{1}-\bar{E}_{2}, \bar{E}_{13}=\bar{E}_{1}-\bar{E}_{3}$. The system is uncoupled in the sense that (5.13) together with (5.11) acts to determine $N_{B}$ and then 5.14 with 5.11 determines $N_{C}$ in principle. The value of $N_{A}$ is taken to start at unity and decrease monotonically to zero. Solutions of (5.13), (5.14) with (5.11) are displayed in Figure 20 as $N_{B}\left(N_{A}\right), N_{C}\left(N_{A}\right), \bar{u}\left(N_{A}\right)$ plots and in Figure 21 in the $N_{B}-N_{C}$ plane. 

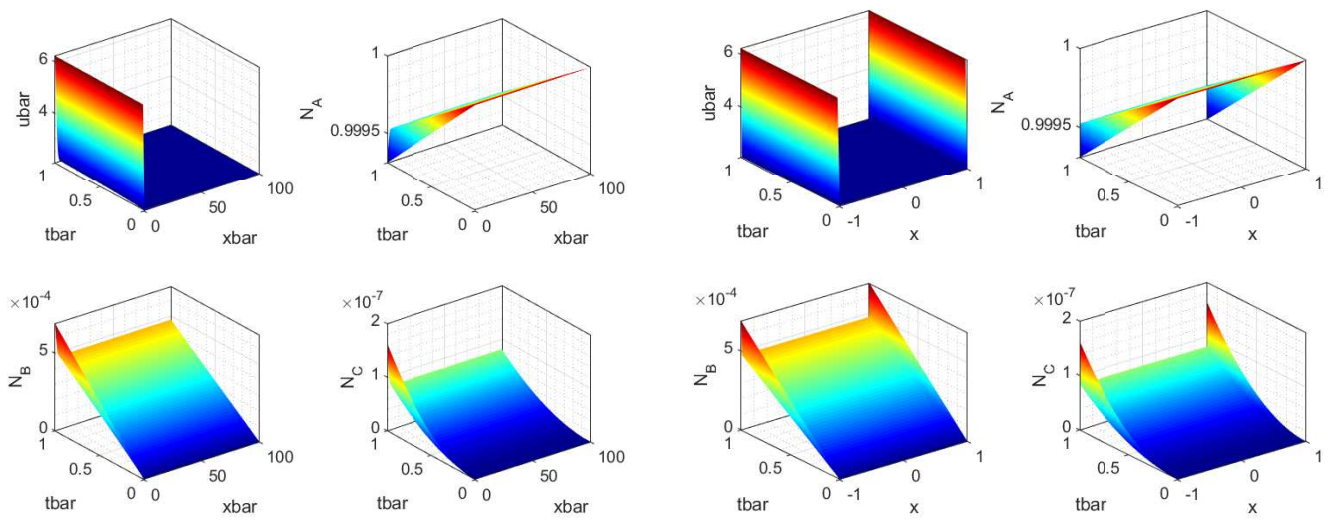

(a) Wall layer solution with mild parameters

(b) Full numerical solution with mild parameters

Figure 18. Finite difference solutions to the full non-asymptotic problem shown (right) and the asymptotic wall problem (left) using the mild parameters in Table 3 . For the non-asymptotic problem we set $\bar{T}_{\max }=1$. In order to compare the solutions directly we multiply the non asymptotic $u$ by $\frac{R}{E}$.

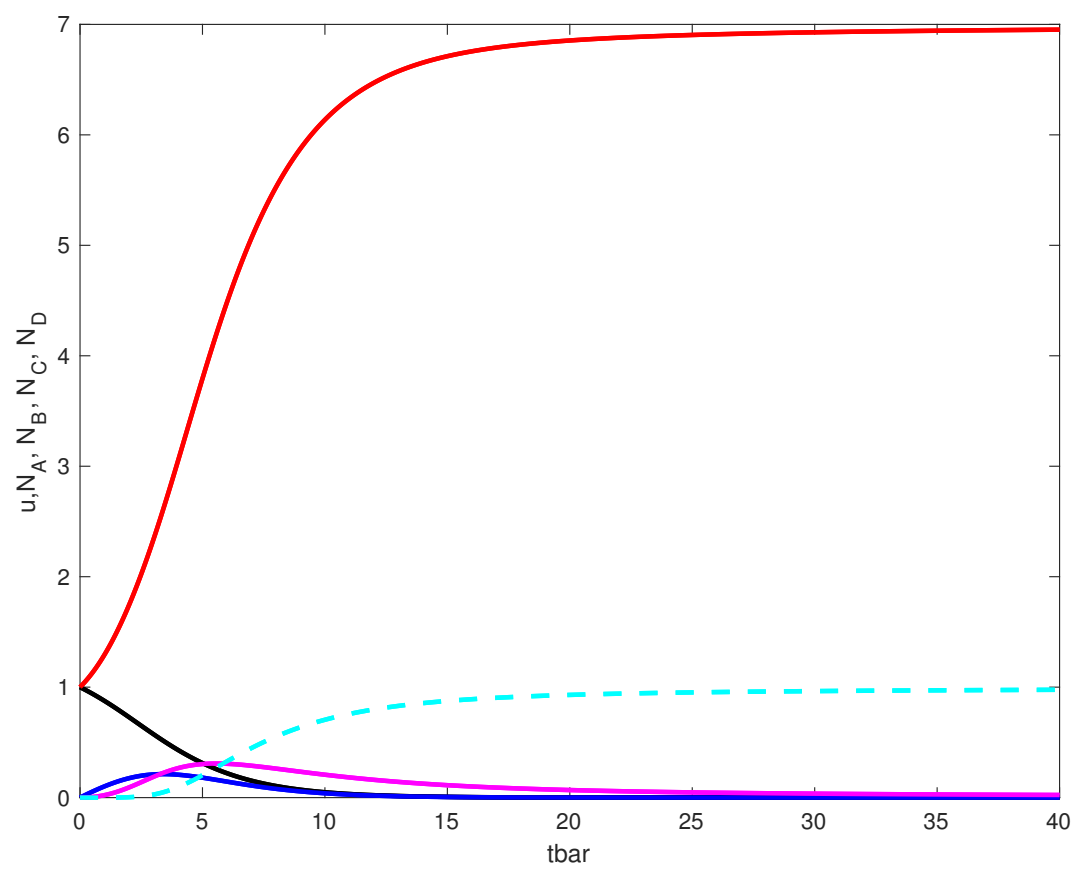

Figure 19. The numerical approximation to Equations 4.5 , (5.6)-(5.8), (5.11). Here the values $\bar{D}=1, d t=0.005, \bar{\Omega}_{1}=1, \bar{\Omega}_{2}=2, \bar{\Omega}_{3}=3, \bar{Z}_{1}=0.5, \bar{Z}_{2}=1, \bar{Z}_{3}=1.5, \bar{E}_{1}=1.5$, $\bar{E}_{2}=1$, and $\bar{E}_{3}=0.5$ have been used. 


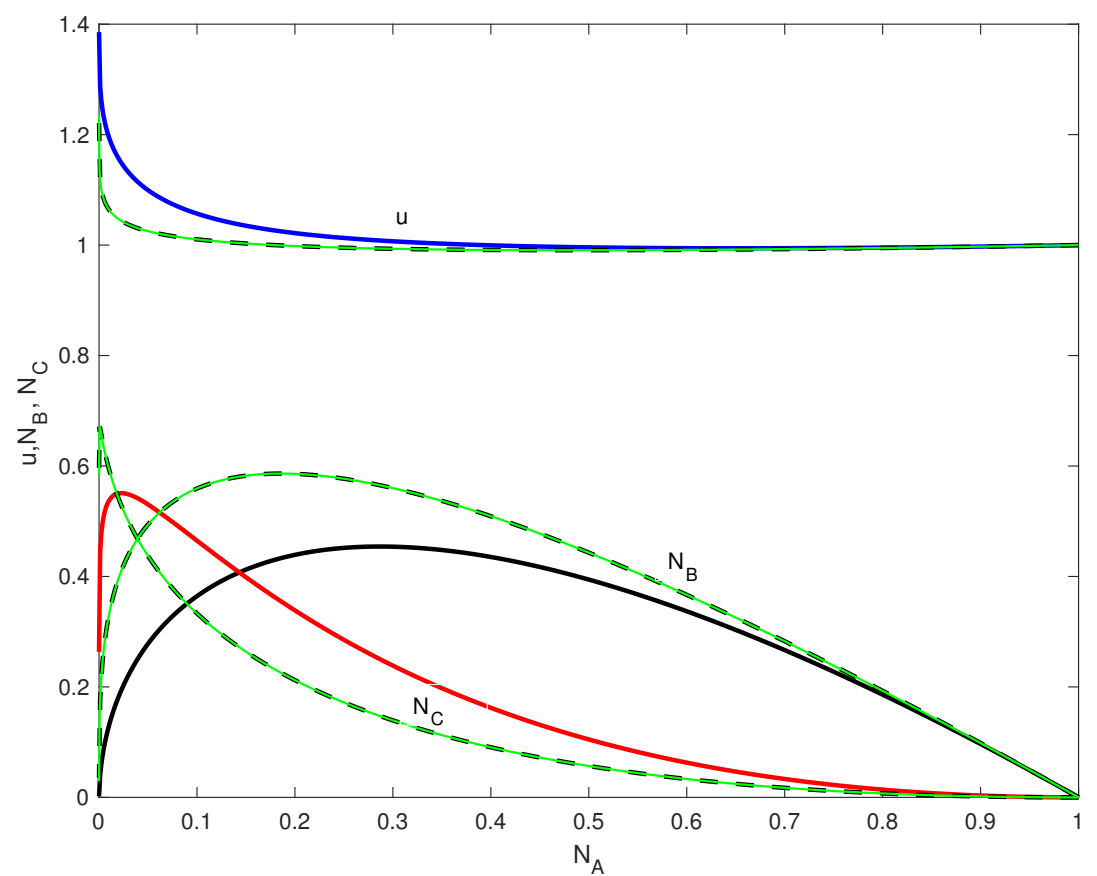

Figure 20. The numerical approximation to Equations (5.11), (5.13), (5.14) for two cases; case 1 is where $\bar{Z}_{21}=0.4$ and $\bar{Z}_{31}=0.16$ displayed by the solid lines (blue, red and black) and case 2 is where $\bar{Z}_{21}=0.2$ and $\bar{Z}_{31}=0.04$ displayed by the green lines. In both cases $\bar{D}=1, \bar{E}_{12}=0.46, \bar{E}_{13}=1, a_{1}=0.5, a_{2}=0.53 a_{3}=0.43$ and $d N_{A}=0.000005$. The dashed lines represent an accuracy check where the effective numerical step $d N_{A}$ is halved.

Subsequently from these solutions $\bar{u}$ can be found from 5.11 and the time-dependence can be found by addressing (5.6) as an equation for $\bar{t}$ as a function of $N_{A}$.

There are seven independent parameters in the phase plane of (5.13), (5.14), namely

$$
\left(a_{1}, a_{2}, a_{3}, \bar{C}, \bar{E}_{12}, \bar{Z}_{21}, \bar{Z}_{31}\right) /\left(\bar{E}_{13}\right),
$$

or combinations thereof, and in the numerical work leading to the above figures we took those parameters to be very mild in value. The alternative of singling out $\bar{E}_{12}$ instead of $\bar{E}_{13}$ would also cover the entire parameter space at issue but in a different way and with equivalent results. We set $\bar{E}_{13}$ equal to unity in the numerical study without loss of generality because here only the ratios of coefficients matter. By contrast, typical numerical values of the parameters for the realistic case mentioned earlier turn out to be

$$
(0.498,0.533,0.427,1,0.462,1.36 e-5,1.070 e-9),
$$

approximately. The small values of $\bar{Z}_{21}, \bar{Z}_{31}$ here suggest consideration of an asymptotic analysis, guided by the insight provided in the single reactant case of Section 3. Taking $\epsilon=\bar{Z}_{21}$ as small and $\bar{Z}_{31}$ as of order $\epsilon^{2}$ we find that there are at least three distinct parts 


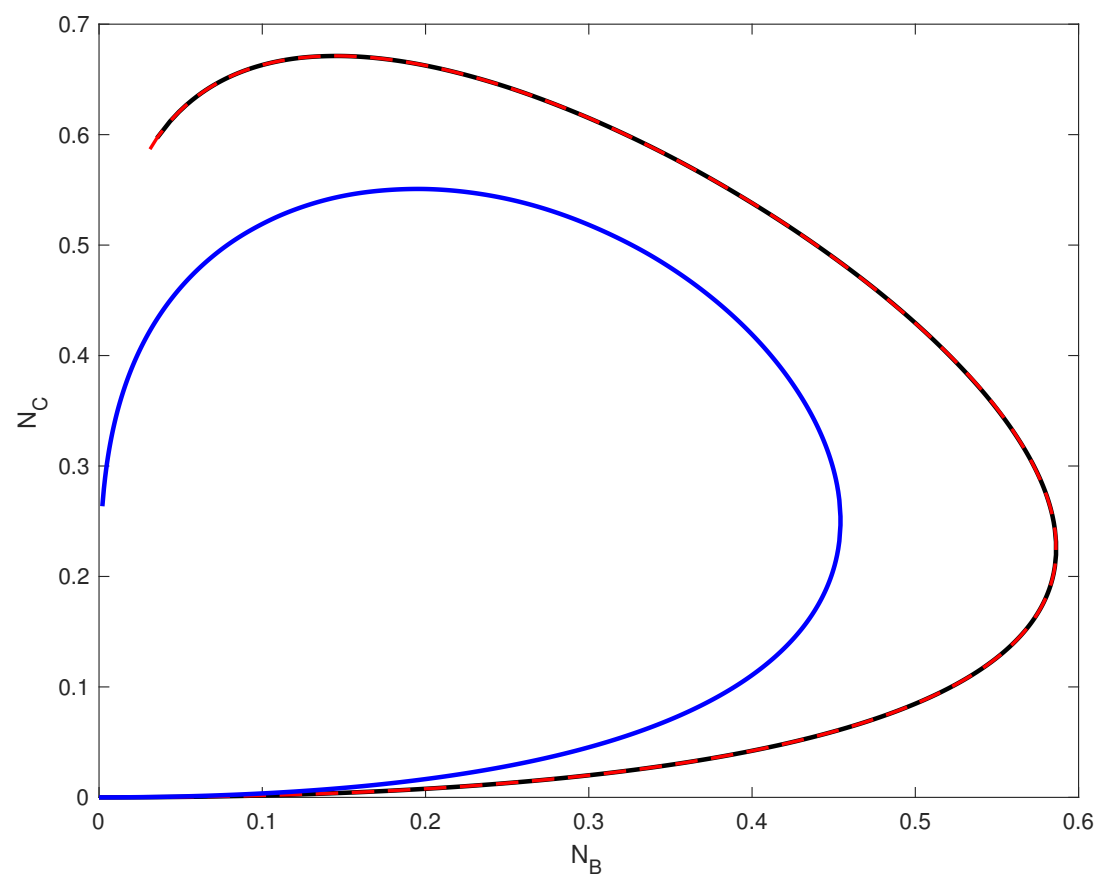

Figure 21. The numerical approximation to Equations 55.11 , (5.13), 5.14 for two cases; case 1 is where $\bar{Z}_{21}=0.4$ and $\bar{Z}_{31}=0.16$ represented by the solid blue line and case 2 is where $\bar{Z}_{21}=0.2$ and $\bar{Z}_{31}=0.04$ represented by the solid red line. In both cases $\bar{D}=1$, $\bar{E}_{12}=0.46, \bar{E}_{13}=1, a_{1}=0.5, a_{2}=0.53 a_{3}=0.43$ and $d N_{A}=0.000005$. Here the dashed black line demonstrates an accuracy check where $d N_{A}$ has been halved.

of the $N_{A}$ range to discuss for 5.13, 5.14). For the majority of the range where $N_{A}$ is of order unity the solution expands as

$$
\begin{aligned}
N_{B} & =N_{B 0}+\epsilon N_{B 1}+\ldots, \\
N_{C} & =\epsilon N_{C 1}+\ldots, \\
\bar{u} & =\bar{u}_{0}+\epsilon \bar{u}_{1}+\ldots,
\end{aligned}
$$

where $\bar{u}_{0}=\left(1-N_{A}\right)\left(a_{1}-a_{2}\right)+\bar{C}$. Substitution into 5.11, 5.13, 5.14 gives successively

$$
\begin{aligned}
N_{B 0} & =1-N_{A}, \\
\frac{d N_{B 1}}{d N_{A}} & =\left(1-N_{A}\right) N_{A}^{-1} \exp \left(\frac{\bar{E}_{12}}{\left(a_{1}-a_{2}\right)\left(1-N_{A}\right)+\bar{C}}\right), \\
\frac{d N_{C 1}}{d N_{A}} & =-\frac{d N_{B 1}}{d N_{A}}, \\
\bar{u}_{1} & =-a_{2} N_{B 1}-a_{3} N_{C 1} .
\end{aligned}
$$

The numerical value of $\bar{u}_{0}$ at $N_{A}=0.5$ is 0.985 , which is suitably close to the computational finding in Figure 20. The trends of 5.20-5.22 are similarly close to those in 
the figure; for example the perturbation in $N_{B}$ about the straight line 5.20$)$ is nearly equal and opposite to the curve of $N_{C}$ indicated by (5.22). The next significant part of the range (apart from a benign region where $N_{A}$ is of order $\epsilon$ ) occurs when $N_{A}$ is exponentially small such that

$$
N_{A}=\exp \left(\frac{-r}{\epsilon}\right) \quad \text { with } N_{B} \text { of } \mathcal{O}(1) .
$$

Here the variable $r$ is typically $\mathcal{O}(1)$. Integration of (5.13) and matching to 5.17) with 5.20 at small $r$, gives us the solution

$$
N_{B}=\exp \left(-c_{1} r\right),
$$

at leading order, where $c_{1}=\exp \left(\frac{\bar{E}_{12}}{a_{1}-a_{2}+C}\right)$ is an $\mathcal{O}(1)$ constant. The $N_{B}$ solution therefore tends to zero as $N_{A}$ tends to zero over this scale (when $r$ tends to infinity), in the form $N_{A}^{c_{1} \epsilon}$, a form which agrees with the balance in (5.13). The corresponding $N_{C}$ however is given by $1-N_{B}$ and so tends to unity then. This leads to the final part of the $N_{A}$ range in which $N_{A}$ is even smaller, specifically

$$
N_{A}=\exp \left(-\frac{s}{\epsilon^{2}}\right) \quad \text { with } N_{C} \text { of } \mathcal{O}(1) .
$$

Here we find that the leading-order solution matching to that of the previous part of the range at small $s$ values is

$$
N_{C}=\frac{c_{5}}{\left(s+c_{5}\right)}
$$

where $c_{5}=\exp \left(-\frac{\bar{E}_{13}}{a_{1}-a_{2}+\bar{C}}\right)$ approximately is an $\mathcal{O}(1)$ positive constant. The $N_{C}$ solution now tends to $0+$ as $N_{A}$ tends to zero over this scale (when $s$ tends to infinity), in the form $c_{5} /\left(-\epsilon^{2} \ln \left(N_{A}\right)\right)$, a form which is in balance with (5.14). The slowness of the approach of $N_{C}$ to zero here compared with that for $N_{B}$ is notable. The trends in (5.24)-(5.27) are consistent with the computational results for $N_{B}, N_{C}$ in Figure 20 at small $N_{A}$ values. The associated time dependence follows from inversion of (5.6); for instance the first part of the $N_{A}$ range ends with

$$
N_{A} \propto \exp \left(-\frac{\bar{Z}_{1} \bar{t}}{c_{6}}\right) \quad \text { as } \bar{t} \rightarrow \infty,
$$

with $c_{6}=\exp \left(-\frac{\bar{E}_{1}}{a_{1}-a_{2}+\bar{C}}\right)$ being a known $\mathcal{O}(1)$ positive constant.

The analytical trends above are consistent with the full computational properties. In addition the result (5.11) implies that the maximum of $\bar{u}$ is $a_{1}+\bar{C}$; in dimensional terms this gives for representative realistic conditions

$$
\bar{u}_{c, \max }=4685 \text { degrees } K,
$$

which again is of potential interest.

\section{Conclusions}

The present work is believed to fill some of the gap in understanding concerned with interaction between burning solid and gas during combustion, through detailed study of 
thermal and reactant properties. Single step reactions have been modelled first using a simple Arrhenius model. A treatment by direct numerical simulation of the fully coupled system involved has been complemented by a treatment founded on asymptotic analysis, with the predictions from the two approaches being found to agree closely in quantitative terms. The model was then extended to include three step reactions and again the combination of simulation and asymptotic analysis was applied, yielding close agreement between results from the full reaction-diffusion problem and those from the asymptotic problem using a mild set of parameters. This verification of the asymptotic model now makes tractable the solution of the reaction diffusion problem over the parameter ranges of real concern without the need for the extremely fine spatial resolution to capture the behaviour near the wall that is needed in direct treatment of the full problem. This has important implications for the application of the model to real problems, where often it is desired to perform parametric studies within a reasonable timescale. Such studies can be greatly hampered by the computational times needed, so this is a significant potential benefit of the work.

This investigation which is based on first principles tends to suggest that scenarios with idealised configurations and analytical modelling can indeed provide significant insight and make it possible to deepen understanding of physical processes arising in real situations. In particular the values of crucial parameters in the models studied turn out to be notably (not to say extremely) small or large, and this leads not only to the helpful comparisons described in the previous paragraph but also to the idea that the asymptotic treatment could act as a viable alternative or supplement to direct numerical simulation. Thus the majority of the spatial domain suffers virtually no diffusion effects, asymptotically, over the main temporal scale of interaction and so the bulk properties of interest are then calculable in a fairly ready fashion. This simplifying treatment adds to the portfolio of methodologies available in the area. In particular we have shown that the asymptotic approach can bring savings in computational resources.

However, there is an issue that may limit the application of the methods of this paper or at least require care in its application. It has been noted that our model is a continuum one and that the thickness of the boundary layer is very small, of the order of $10^{-12}$ times the macroscopic dimension of the explosive perpendicular to the surface. Under circumstances where the explosive may be treated as entirely homogeneous, such as for a single macroscopic crystal of explosive, this poses no difficulties and the model may be directly applied. However, many high explosives contain small crystals of the order of $10-50 \mu \mathrm{m}$ embedded in a polymer binder. Here the boundary layer is but a tiny fraction of the crystal size. There may therefore be materials of different conductivities crossing the whole or part of the thickness of the boundary layer. It may be possible to use volume averaged properties effectively with the analysis we have presented, suitably adjusting the parameters we have used in a straightforward manner. However, we acknowledge we may instead need to consider a refinement of our analysis, possibly addressing threedimensional effects, at the mesoscopic rather than continuum scale, to reach a physically realistic model. This issue, unforeseen at the outset of the research, may well have very important implications for understanding the physics of burning.

A final point here, particularly relevant to the point just discussed, is that the model of interaction evolving between thermal diffusion effects and a number of reactants has 
been considered for one spatial dimension only. The present approach, especially the asymptotic treatment but to some extent the direct treatment also, indicates that reliable predictions for two- and three-dimensional configurations should be quite possible [33]. This is simply because diffusion is virtually negligible (see previous paragraph) and so the interaction process is pointwise, unaffected by the containing geometry. If we accept that then the task of prediction in both the two- and the three-dimensional cases becomes a relatively simple calculation. The potential for savings in computational resources as mentioned above is even greater for two-and three-dimensional cases, which, of course, are prevalent in reality. We would add that the application of asymptotics helps the modeller gain deeper insights into the physics of the modelled situation. It can provide useful independent verification checks to give greater confidence in past and future model predictions, where direct numerical simulations are applied.

\section{Acknowledgements}

Our thanks go to our colleagues for their interest and questions and to AWE and EPSRC for financial support of SS through an industrial CASE award (EPSRC grant reference $\mathrm{EP} / \mathrm{R} 512138 / 1)$. We wish to thank a referee for helpful comments.

\section{References}

[1] P. W. Cooper and S. R. Kurowski, Introduction to the Technology of Explosives. 1996.

[2] P. W. Cooper, Explosives engineering. 1996.

[3] K. W. Morton and D. Mayers, Numerical Solution of Partial differential Equations. 2005.

[4] M. Ã-zisik, M. Özısık, and M. Özışık, Heat Conduction. Wiley-Interscience publication, Wiley, 1993.

[5] J. M. Groocock, "Griffiths and groocock : The burning to detonation of solid explosives," Journal of the Chemical Society., no. 4154, 1958.

[6] M. L. Blanford, "Jas3da multi-strategy iterative code for solid mechanics analysis," Sandia National Laboratories., 1998.

[7] M. Ward, S. Son, and M. Brewster, "Steady deflagration of hmx with simple kinetics: A gas phase chain reaction model," Combustion and Flame, 1997.

[8] C. Tarver, T. Tran, and R. Whipple, "Thermal decomposition of pentaerythritol tetranitrate," Propellants, Explosives, Pyrotechnics, vol. 28, no. 4, pp. 189-193, 2003.

[9] A. L. Nichols, "Improving the material response for slow heat of energetic materials," Lawrence Livermore National Laboratory (LLNL).

[10] J. Bear, Dynamics of fluids in porous media. New York : American Elsevier, 1972.

[11] M. Baer and J. Nunziato, "A two-phase mixture theory for the deflagration-to-detonation transition (ddt) in reactive granular materials," International Journal of Multiphase Flow, vol. 12 , no. 6 , pp. $861-889,1986$.

[12] D. Breshears, "One dimensional time-to-explode (odtx) in hmx spheres," Los Alamos National Lab. Technical Report.

[13] P. C. Hsu, G. Hust, M. Howard, and J. L. Maienschein, "The odtx system for thermal ignition and thermal safety study of energetic materials," Lawrence Livermore National Laboratory (LLNL).

[14] F. Williams, Combustion Theory. Addison-Wesley series in engineering sciences, AddisonWesley Publishing Company, 1965.

[15] J. J. Yoh, M. A. McClelland, J. L. Maienschein, A. L. Nichols, and C. M. Tarver, "Simulating 
thermal explosion of octahydrotetranitrotetrazine-based explosives: Model comparison with experiment," Journal of Applied Physics, vol. 100, no. 7, p. 073515, 2006.

[16] S. Bullett, T. Fearn, and F. Smith, Advanced Techniques in Applied Mathematics. WORLD SCIENTIFIC (EUROPE), 2016.

[17] J. Curtis, "A new analytical model of the one-dimensional time to explosion experiment," Unpublished AWE Report, October 2018.

[18] A. C. Victor, "Simple calculation methods for munitions cookoff times and temperatures," Propellants, Explosives, Pyrotechnics, vol. 20, no. 5, pp. 252-259, 1995.

[19] C. Tarver, R. McGuire, E. Lee, E. Wrenn, and K. Brein, "The thermal decomposition of explosives with full containment in one-dimensional geometries," Symposium (International) on Combustion, vol. 17, no. 1, pp. 1407 - 1413, 1979. Seventeenth Symposium (International) on Combustion.

[20] P. E. Luebcke, P. M. Dickson, and J. E. Field, "Deflagration-to-Detonation Transition in Granular Pentaerythritol Tetranitrate," J. Appl. Phys., vol. 79, no. 7, pp. 3499-3503, 1996.

[21] R. R. McGuire and C. M. Tarver, "Chemical Decomposition Models for the Thermal Explosion of Confined HMX, TATB, RDX, and TNT Explosives," Symposium on Detonation, no. June, p. 11, 1981.

[22] J. Bdzil and S. Son, "Engineering models of deflagration-to-detonation transition," Los Alamos National Laboratory, LA-12794-MS, 1995.

[23] M. Becker, Heat Transfer Analysis and Design Problems. Boston, MA: Springer US, 1986.

[24] E. Catalano, R. McGuire, E. Lee, E. Wrenn, D. Ornellas, and J. Walton, "Thermal decomposition and reaction of confined explosives. [tnt, tatb, lx-04, lx-10]," Proceedings of Sixth Symposium (International) on Detonation, pp. 214-222, 1976.

[25] M. Hobbs, M. Baer, and R. Gross, "A constitutive mechanical model for energetic materials.," Proceedings of the 20th International Pyrotechnics Seminar, 061994.

[26] S. Vyazovkin, A. K. Burnham, J. M. Criado, L. A. Pérez-Maqueda, C. Popescu, and N. Sbirrazzuoli, "ICTAC Kinetics Committee recommendations for performing kinetic computations on thermal analysis data," Thermochimica Acta, vol. 520, no. 1-2, pp. 1-19, 2011.

[27] D. A. Anderson, Computational fluid mechanics and heat transfer. Series in computational methods in mechanics and thermal sciences, Washington: Hemisphere Pub. Corp., 1984.

[28] D. Anderson, J. Tannehill, and R. Pletcher, "Computational fluid mechanics and heat transfer,"

[29] M. E. Brown, "The Prout-Tompkins rate equation in solid-state kinetics," Thermochimica Acta, vol. 300, no. 1-2, pp. 93-106, 1997.

[30] F. Morrison, "Transient gas flow in a porous column," IEEEC Fundamentals, vol. 11, pp. 191197,1972

[31] H. S. Carslaw, J. C. Jaeger, and J. E. Morral, Conduction of Heat in Solids, Second Edition, vol. 108. 1986.

[32] S. Said, "Analytical and numerical modelling of the combustion of explosives," in preparation.

[33] J. P. Curtis, J. E. Reaugh, and C. A. Handley, "On Modelling the Evolution of Specific Surface Area in the Burning of Explosives," Proceedings of the Forty-Fourth International Pyrotechnics Seminar, 2019. 